TRANSACTIONS OF THE

AMERICAN MATHEMATICAL SOCIETY

Volume 358, Number 12, December 2006, Pages 5565-5585

S 0002-9947(06)03915-8

Article electronically published on July 20, 2006

\title{
VARIETIES WITH SMALL DISCRIMINANT VARIETY
}

\author{
ANTONIO LANTERI AND ROBERTO MUÑOZ
}

\begin{abstract}
Let $X$ be a smooth complex projective variety, let $L$ be an ample and spanned line bundle on $X, V \subseteq H^{0}(X, L)$ defining a morphism $\phi_{V}: X \rightarrow \mathbb{P}^{N}$ and let $\mathcal{D}(X, V)$ be its discriminant locus, the variety parameterizing the singular elements of $|V|$. We present two bounds on the dimension of $\mathcal{D}(X, V)$ and its main component relying on the geometry of $\phi_{V}(X) \subset \mathbb{P}^{N}$. Classification results for triplets $(X, L, V)$ reaching the bounds as well as significant examples are provided.
\end{abstract}

\section{INTRODUCTION}

Let $L$ be an ample line bundle on a smooth complex projective variety $X$ of dimension $n$. Assume that $L$ is spanned by a vector space $V \subseteq H^{0}(X, L)$ of global sections of dimension $N+1$ which defines a map $\phi_{V}: X \rightarrow \mathbb{P}^{N}$. The discriminant locus $\mathcal{D}(X, V)$ consists of the singular elements of the linear system $|V|$ defined by $V$. When $L$ is very ample and $\phi_{V}$ is an embedding, $\mathcal{D}(X, V)$ is nothing else but the dual variety $X^{\vee} \subset \mathbb{P}^{N \vee}$ of the projective variety $X \subset \mathbb{P}^{N}$. In [LPS1] a careful study of the discriminant locus $\mathcal{D}(X, V)$ of a triplet $(X, L, V)$ is developed, giving some bounds for its dimension and constructing a sort of stratification of $\mathcal{D}(X, V)$ by means of the jumping sets $\mathcal{J}_{i}(V)(i=1, \ldots, n)$ which measure the deviation from $\phi_{V}$ to be an embedding. In particular, the jumping sets are related with the Chern classes of the first jet bundle $J_{1}(L)$.

Bertini's Theorem implies $\operatorname{dim}(\mathcal{D}(X, V)) \leq N-1$, and it is an interesting problem to classify triplets $(X, L, V)$ for which $\mathcal{D}(X, V)$ is not a hypersurface. A natural concept of defect appears: $\operatorname{def}(X, V)=N-1-\operatorname{dim}(\mathcal{D}(X, V))$. We call these triplets varieties with small discriminant variety (or equivalently positive defect varieties). In the classical setting, that is, when $\phi_{V}$ is an embedding, the problem of classification of smooth varieties with small dual variety has been faced in different ways. The following are some results related to this problem:

(i) From Zak's Theorem on tangencies [Z, Cor. 1.8] it holds that $\operatorname{dim}(X) \leq$ $\operatorname{dim}\left(X^{\vee}\right)$, and so $\operatorname{dim}(X)=\operatorname{dim}\left(X^{\vee}\right)-p$, with $p \in \mathbb{Z}$ and $p \geq 0$. In E1 the case $p=0$ is studied, giving a complete classification under the hypothesis $\operatorname{dim}(X) \leq$ $2 N / 3$. In M1 a complete classification result is shown for the case $p=1$ under the same hypothesis. Moreover, a finiteness result is shown for any $p$. In [M3] a partial result of classification is done for the case $p=2$.

Received by the editors February 17, 2004 and, in revised form, November 26, 2004.

2000 Mathematics Subject Classification. Primary 14J40, 14N05, 14C20; Secondary 14F05, $14 \mathrm{M} 99$.

Key words and phrases. Complex projective variety, duality, defect, discriminant loci. 
(ii) Adjunction theory is used in BFS, Th. 1.2] to prove a structure theorem for positive defect varieties. Precisely, it is shown that a positive defect variety is a fibration over a normal variety such that the fibres are positive defect Fano varieties.

(iii) From the biduality theorem (that is, $X^{\vee \vee} \cong X$ under the natural identification between $\mathbb{P}^{N \vee \vee}$ and $\left.\mathbb{P}^{N}\right)$ it holds that $\operatorname{def}(X, V)$ is precisely the dimension of the singular locus of a general singular hyperplane section. This means that $\operatorname{def}(X, V) \leq n$ with equality only if $X=\mathbb{P}^{n}$. In fact, by topological methods or by studying the singularities of the general tangent hyperplane section [E1], it is shown that $\operatorname{def}(X, V)$ has the same parity as $\operatorname{dim}(X)$ (usually called Landman's parity theorem). Hence $\operatorname{def}(X, V)=n-2 p$, with $p \in \mathbb{Z}$ and $p \geq 0$. The case $p=0$ corresponds to $X=\mathbb{P}^{n}$. The case $p=1$ corresponds to scrolls over curves [LS1], E2. The case $p=2$ consists of scrolls over surfaces, the embedding of Plücker of the grassmannian of lines in $\mathbb{P}^{4}, G(1,4) \subset \mathbb{P}^{9}$, and its hyperplane sections E1, E2]. Also, when $\operatorname{dim}(X)$ is small, some more results of classification can be found in LS2, [BFS and M2. For a nice survey on the subject of dual varieties we can refer to $[\mathrm{T}]$.

Coming back to the general setting, it is clear that the dual variety of $\phi_{V}(X) \subseteq$ $\mathbb{P}^{N}$ is contained in $\mathcal{D}(X, V)$. Then it is natural to look for bounds on $\operatorname{dim}(\mathcal{D}(X, V))$ like those of (i) and (iii) and for structure theorems in the spirit of (ii) using what we know about $\phi_{V}(X)^{\vee} \subset \mathbb{P}^{N \vee}$. In Section 1, using the generalized statement of Zak's theorem on tangencies, we get a first bound on $\operatorname{dim}(\mathcal{D}(X, V))$, precisely $\operatorname{dim}(\mathcal{D}(X, V)) \geq n-\operatorname{dim}\left(\operatorname{Sing}\left(\phi_{V}(X)\right)\right)-1$, and we classify triplets $(X, L, V)$ for which the previous inequality is in fact an equality. In Theorem (1.10) we show that either $\phi_{V}(X)$ is a smooth hypersurface, or $L$ is very ample and $\phi_{V}$ an isomorphism (i.e., we are in the classical case), or $\phi_{V}(X)$ is the Segre embedding of $\mathbb{P}^{1} \times \mathbb{P}^{n-1}$ in $\mathbb{P}^{2 n-1}$. To prove this we need a result of classification of singular projective varieties with small dual variety, similar to (i). The precise problem is posed in Problem (1.4), and it is of interest by itself: to classify projective varieties $X \subset \mathbb{P}^{N}$ such that $\operatorname{dim}\left(X^{\vee}\right)=\operatorname{dim}(X)-\operatorname{dim}(\operatorname{Sing}(X))-1$. We prove in Theorem (1.6) that they are either smooth varieties with $\operatorname{dim}(X)=\operatorname{dim}\left(X^{\vee}\right)$ or cones over those varieties. In Section (1.12) some considerations about the next case, that is, triplets $(X, L, V)$ verifying $\operatorname{dim}(\mathcal{D}(X, V))=\operatorname{dim}(X)-\operatorname{dim}(\operatorname{Sing}(X))$, are provided.

A natural way to produce examples of positive defect triplets $(X, L, V)$ is to consider ramified cyclic coverings of smooth positive defect varieties. For this kind of construction only the first jumping set is non-empty. Then $\mathcal{D}(X, V) \backslash \phi_{V}(X)^{\vee}$ comes from the ramification locus of $\phi_{V}$. In Section 2 we study completely this situation and prove a structure theorem, Theorem $(2.6)$, showing that $\phi_{V}(X)$ is a fibration over a normal variety $Z$ whose fibres are Fano varieties and $X$ is a Fano fibration over a normal variety $T$ with the same fibres as $\phi_{V}(X)$.

In [LPS1, Th. 2.8] it is shown that $\operatorname{def}(X, V) \leq \operatorname{dim}(X)$ and equality holds when $(X, L, V)=\left(\mathbb{P}^{n}, \mathcal{O}_{\mathbb{P}^{n}}(1), H^{0}\left(\mathbb{P}^{n}, \mathcal{O}_{\mathbb{P}^{n}}(1)\right)\right)$. It is also shown that $\operatorname{def}(X, V)$ cannot be equal to $\operatorname{dim}(X)-1$. In the same paper [LPS1, Conj. 2.11] it is conjectured that if $\operatorname{def}(X, V)>0$, then $X$ is swept out by linear spaces. This conjecture holds when $\phi_{V}$ is generically one-to-one as a consequence of [LPS2, Cor. 2.5]. In fact the linear spaces in $X$ come from the linear spaces in $\phi_{V}(X)$ corresponding to the singularities of its general tangent hyperplanes. In the study of varieties with small dual variety these linear spaces are crucial. Then, at least when $\phi_{V}$ is generically 
one-to-one, or always if the quoted conjecture holds, it seems interesting to deal with them. Define the zero defect as $\operatorname{def}_{0}(X, V)=N-1-\operatorname{dim}\left(\phi_{V}(X)^{\vee}\right)$. Since $\phi_{V}(X)^{\vee} \subseteq \mathcal{D}(X, V)$ then $\operatorname{def}_{0}(X, V) \geq \operatorname{def}(X, V)$. Some questions arise about the zero defect. First one is to know which values $\operatorname{def}_{0}(X, V)$ can reach. By the biduality theorem $0 \leq \operatorname{def}_{0}(X, V) \leq n$; moreover $\operatorname{def}_{0}(X, V)=n$ if and only if $\phi_{V}(X)=\mathbb{P}^{n}$. In particular if $\phi_{V}$ is generically one-to-one, then $\operatorname{def}_{0}(X, V)=n$ if and only if $(X, L, V)=\left(\mathbb{P}^{n}, \mathcal{O}_{\mathbb{P}^{n}}(1), H^{0}\left(\mathbb{P}^{n}, \mathcal{O}_{\mathbb{P}^{n}}(1)\right)\right)$. In Example (3.2) we construct examples $(X, L, V)$ for which any possible value is attained, satisfying moreover that $\phi_{V}$ is generically one-to-one. Second one is to classify the extremal case of triplets $(X, L, V)$ verifying the equality $\operatorname{def}_{0}(X, V)=n-1$, and the quasi-extremal case, those verifying the equality $\operatorname{def}_{0}(X, V)=n-2$. In Propositions (4.2) and (4.5) we establish this classification under the additional hypothesis that $\phi_{V}$ is generically one-to-one. They are scrolls over curves and surfaces, but $\phi_{V}$ can map $X$ onto a developable variety. The proof of the classification results relies on the fact that in both situations $X$ is swept out by linear spaces. Let us observe that if $\operatorname{def}(X, V)=n-2$, then $\operatorname{def}_{0}(X, V) \geq n-2$, so this is a first step in the classification of triplets $(X, L, V)$ with $\operatorname{def}(X, V)=n-2$.

During the preparation of this paper the first author was partially supported by the MIUR of the Italian Government in the framework of the PRIN "Geometry on Algebraic Varieties" (Cofin 2002). The research of the second author was partially supported by the MCYT projects BFM2000-0621 and BFM2003-03971. Both authors acknowledge the support provided by azione integrata IT200 at an early stage of this research.

\section{BACKGRound MATERIAL}

Throughout the paper we will work over the field of complex numbers, and we will use standard notation and terminology in algebraic geometry.

(0.0) Let $X$ be a smooth complex projective variety of dimension $n \geq 1$ and let $L$ be an ample line bundle on $X$, spanned by a vector subspace $V \subseteq H^{0}(X, L)$ of dimension $N+1$. Let $\phi_{V}: X \rightarrow \mathbb{P}^{N}$ be the morphism associated to $V$.

Since the term scroll is used with different meanings in the literature, let us write the following

(0.1) Definition. We say that a pair $(X, L)$ as in Section $(0.0)$ is a scroll over a variety $Y$ with $\operatorname{dim}(Y)=m<n$, if there exists a surjective morphism $p: X \rightarrow Y$ such that $\left(f,\left.L\right|_{f}\right)=\left(\mathbb{P}^{n-m}, \mathcal{O}_{\mathbb{P}^{n-m}}(1)\right)$ for every fibre $f$ of $p$.

As in [LPS1, Sec. 1] we can consider the discriminant locus $\mathcal{D}=\mathcal{D}(X, V)$ parameterizing all singular elements of the linear system $|V|$ defined by the sections in $V$. In general $\mathcal{D}$ consists of several irreducible components. If $N>n$ the main component, which we denote by $\mathcal{D}_{0}$, corresponds to the dual variety $\phi_{V}(X)^{\vee}$ of $\phi_{V}(X) \subset \mathbb{P}^{N}$ under the identification of $|V|$ with the dual projective space $\mathbb{P}^{N \vee}$. However, extra components may occur. Define the $i$-th jumping set $\mathcal{J}_{i}(X, V)$ as the locus of points of $X$ such that the rank of the differential of the map $\phi_{V}$ is less than or equal to $n-i$, that is, $\mathcal{J}_{i}(X, V)=\left\{x \in X: \operatorname{rk}\left(d \phi_{V}(x)\right) \leq n-i\right\}$. It is clear that $X \supseteq \mathcal{J}_{1}(X, V) \supseteq \cdots \supseteq \mathcal{J}_{n}(X, V)$. If $\mathcal{J}_{1}(X, V)$, the locus where $\phi_{V}$ ramifies, or some other jumping set $(i \geq 2)$ is non-empty, then some extra 
components appear. Examples describing the various components of $\mathcal{D}$ and some pathologies which may occur for them can be found in [LPS1, Sec. 1], [LPS2, Sec. 4]. To be precise, we define $\mathcal{D}_{0}$ in the following way. Let $U=X \backslash \mathcal{J}_{1}(X, V)$ and consider the bundle surjection $\mu: V \times\left.\mathcal{O}_{U} \rightarrow J_{1}(L)\right|_{U}$ given by evaluation of 1-jets of sections in $V$. Denote by $\mathcal{K}$ the dual of the kernel of $\mu$. Then $\mathcal{D}_{0}$ is the image of the closure of $\mathbb{P}(\mathcal{K})$ via the second projection of $X \times|V|$. Note that if $D \in|V-2 x|$ for $x \in U$, then certainly $D \in \mathcal{D}_{0}$. For $i \geq 1$ let $\mathcal{D}_{i}$ be the union of the irreducible components $\mathcal{D}_{i j}$ associated to the irreducible components of $\overline{\mathcal{J}_{i}(X, V) \backslash \mathcal{J}_{i+1}(X, V)}$. For every $i=0, \ldots, n$ we $\operatorname{set} \operatorname{def}_{i}(X, V)=N-1-\operatorname{dim} \mathcal{D}_{i}$. Clearly $\operatorname{def}_{0}(X, V)$ is simply the classical dual defect of the projective variety $\phi_{V}(X) \subset \mathbb{P}^{N}$, that is, $\operatorname{def}\left(\phi_{V}(X)\right)=N-1-\operatorname{dim}\left(\phi_{V}(X)^{\vee}\right)$. The behavior of the $\operatorname{def}_{i}$ for $i \geq 1$ will be studied elsewhere. We set $\operatorname{def}(X, V)=N-1-\operatorname{dim}(\mathcal{D}(X, V))$. We write $\operatorname{def}(X, L)$ $\left(\operatorname{def}_{i}(X, L)\right)$ to mean that $V=H^{0}(X, L)$. Note that

$$
\operatorname{def}(X, V)=\min _{0 \leq i \leq n}\left\{\operatorname{def}_{i}(X, V)\right\}
$$

by definition. Hence, if $\operatorname{def}(X, V)>0$, then $\operatorname{def}_{i}(X, V)>0$ for every $i$. Moreover, since $\operatorname{dim} \mathcal{D}_{i}=\max _{j}\left\{\operatorname{dim} \mathcal{D}_{i j}\right\}$ for $i \geq 1$, it is clear that if $\operatorname{def}_{i}(X, V)>0$, then $\operatorname{dim} \mathcal{D}_{i j}<N-1$ for every $j$.

(0.0.2) Let us recall here [LPS1, Th. 2.8] that $\operatorname{def}(X, V) \leq n$ with equality if and only if $(X, L, V)=\left(\mathbb{P}^{n}, \mathcal{O}_{\mathbb{P}^{n}}(1), H^{0}\left(\mathbb{P}^{n}, \mathcal{O}_{\mathbb{P}^{n}}(1)\right)\right)$.

To clarify concepts and because it is going to be of interest in upcoming sections, let us give an example.

(0.2) Example. Let $X$ be a Del Pezzo surface with $K_{X}^{2}=1$, let $L=-2 K_{X}$ and let $V=H^{0}(X, L)$. As is known, $\left|-K_{X}\right|$ is a pencil having a single base point $x^{*}$, while $L$ is ample and spanned, and $\phi_{V}: X \rightarrow \mathbb{P}^{3}$ exhibits $X$ as a double cover of a quadric cone $\Gamma \subset \mathbb{P}^{3}$, branched at the vertex $v=\phi_{V}\left(x^{*}\right)$ and along the transverse intersection $B$ with a cubic surface. The jumping sets of $(X, V)$ are $\mathcal{J}_{1}(X, V)=$ $\phi_{V}^{-1}(B) \cup\left\{x^{*}\right\}$ and $\mathcal{J}_{2}(X, V)=\left\{x^{*}\right\}$ (see [LPS1, Ex. 1.8, iii)]). Thus $\mathcal{D}(X, V)=$ $\mathcal{D}_{0} \cup \mathcal{D}_{1} \cup \mathcal{D}_{2}$, where, under the identification of $|V|$ with $\mathbb{P}\left(H^{0}\left(\Gamma, \mathcal{O}_{\Gamma}(1)\right)\right): \mathcal{D}_{0}=\Gamma^{\vee}$ is the dual variety of $\Gamma$ and $\mathcal{D}_{1}=B^{\vee}$ is the dual variety of $B$. Moreover, $\mathcal{D}_{2}=$ $\left\{C_{1}+C_{2}\left|C_{i} \in\right|-K_{X} \mid\right\}=\left|-K_{X}\right|^{(2)} \cong \mathbb{P}^{2}$ can be regarded as the star of planes of $\mathbb{P}^{3}$ through the vertex $v$. Since $\Gamma^{\vee}$ is a conic while $\mathcal{D}_{1}, \mathcal{D}_{2}$ are surfaces, we get $\operatorname{def}_{0}(X, V)=1, \operatorname{def}_{1}(X, V)=\operatorname{def}_{2}(X, V)=0$.

\section{First Bound ON THE Dimension OF THE DISCRIMINANT LOCUS}

Let us recall the following theorem of Zak.

(1.1) Theorem ([Z, Cor. 2.5]). Let $X \subset \mathbb{P}^{N}$ be a non-degenerate non-linear projective variety and call $b$ the dimension of the singular locus of $X$. Then $\operatorname{dim}\left(X^{\vee}\right) \geq$ $n-b-1$.

We have to exclude the possibility of $X$ being linear because then $X^{\vee}=\emptyset$. Since $\operatorname{dim}(\mathcal{D}(X, V)) \geq \operatorname{dim}\left(\mathcal{D}_{0}(X, V)\right)$, we can use (1.1) to get

(1.2) First Bound Lemma. Let $(X, L, V)$ be a triplet as in Section (0.0) such that $\phi_{V}(X) \subset \mathbb{P}^{N}$ is not linear. Then $\operatorname{dim}(\mathcal{D}(X, V)) \geq n-1-\operatorname{dim}\left(\operatorname{Sing}\left(\phi_{V}(X)\right)\right)$. 
In particular, when $L$ is very ample and $\phi_{V}$ is an embedding, we have the classical bound $\operatorname{dim}\left(X^{\vee}\right) \geq \operatorname{dim}(X)$. In general, if equality holds in the bound of Lemma $(1.2)$, then

$$
\operatorname{dim}\left(\mathcal{D}_{0}(X, V)\right)=\operatorname{dim}\left(\phi_{V}(X)^{\vee}\right)=n-\operatorname{dim}\left(\operatorname{Sing}\left(\phi_{V}(X)\right)\right)-1 .
$$

It is natural to consider the following.

(1.3) Problem. The classification of $(X, L, V)$ as in Section (0.0) such that $\phi_{V}(X)$ is not linear and $\operatorname{dim}(\mathcal{D}(X, V))=n-1-\operatorname{dim}\left(\operatorname{Sing}\left(\phi_{V}(X)\right)\right)$.

In fact there are examples (the bound is sharp).

(1.3.1) Examples. (i) Ein varieties, that is, smooth varieties $(b=-1) X \subset \mathbb{P}^{N}$ of codimension bigger than one, with $\operatorname{dim}(X)=\operatorname{dim}\left(X^{\vee}\right)$. Let us recall here that, with the hypothesis $\operatorname{dim}(X) \leq 2 N / 3$, Ein classified smooth projective varieties $X \subset \mathbb{P}^{N}$ with $\operatorname{dim}\left(X^{\vee}\right)=\operatorname{dim}(X)$. This justifies the name of Ein varieties. They are the following, [E1, Th. 4.5]: the Segre embedding $\mathbb{P}^{1} \times \mathbb{P}^{n-1} \subset \mathbb{P}^{2 n-1}$; the Plücker embedding of the grassmannian of lines of $\mathbb{P}^{4}, G(1,4) \subset \mathbb{P}^{9}$; the spinor variety $S_{4} \subset \mathbb{P}^{15}$.

(ii) Consider a degree $k$ base-point-free pencil on a smooth curve $C$ of genus $g$ and the induced map $f_{k}: C \rightarrow \mathbb{P}^{1}$. Let $X=C \times \mathbb{P}^{n-1}$. Defining $\phi_{V}$ as the composition of the $k$-to-one map $f_{k} \times I d: X \rightarrow \mathbb{P}^{1} \times \mathbb{P}^{n-1}$ (where $I d$ stands for the identity of $\mathbb{P}^{n-1}$ ) with the Segre embedding into $\mathbb{P}^{2 n-1}$, we get a triplet as in Section (0.0). In this setting, $\mathcal{D}_{0}=\left(\mathbb{P}^{1} \times \mathbb{P}^{n-1}\right)^{\vee}$, and $\mathcal{D}_{1}$ is a union of (in general $2 g+2 k-2) \mathbb{P}^{n-1}$ 's. Since $\operatorname{Sing}\left(\phi_{V}(X)\right)=\emptyset$ we reach the bound.

By (1.2.1), for any $(X, L, V)$ as in Problem $(1.3), \phi_{V}(X) \subset \mathbb{P}^{N}$ is a variety reaching the bound in Theorem (1.1). Then we must first consider the following.

(1.4) Problem. Classify non-degenerate non-linear projective varieties $X \subset \mathbb{P}^{N}$ for which $\operatorname{dim}\left(X^{\vee}\right)=n-\operatorname{dim}(\operatorname{Sing}(X))-1$.

For this purpose we need the following lemma.

(1.5) Lemma. Let $X \subset \mathbb{P}^{N}$ be a non-degenerate variety with isolated singularities such that $\operatorname{dim}\left(X^{\vee}\right)<N-1$. Then either $X \subset \mathbb{P}^{N}$ is a cone, or the general contact locus does not meet the set of singularities of $X$.

Proof. Let us recall that for a general point $x$ in $X$ and for a general hyperplane $H$ tangent to $X$ at $x$, it holds that $R=\operatorname{Sing}(X \cap H)$ is, by biduality theorem, a linear space (called the contact locus of $H$ with $X$ ) of positive dimension $k=$ $N-1-\operatorname{dim}\left(X^{\vee}\right)$. Let $\left\{p_{1}, \ldots, p_{n}\right\} \subset X$ be the set of singular points of $X$. Take $U \subset X^{\vee}$ the open set of smooth points of the dual variety and define the map $f: U \rightarrow G(k, N)$ sending a general tangent hyperplane to its contact locus. Let us write $Z$ for the closure of $f(U)$. If $Z=Z_{1} \cup \cdots \cup Z_{n}$ where $Z_{i}=\left\{R \in Z: p_{i} \in R\right\}$, then, since $Z$ is irreducible, there exists $i \in\{1, \ldots, n\}$ such that $Z=Z_{i}$. This means that for a general $x \in X$ the line $\left\langle x, p_{i}\right\rangle$ is contained in $X$ and then $X$ is a cone. Let us note that if $i$ is not unique, then $X$ is a cone over a linear space of positive dimension, contradicting $\operatorname{dim}(\operatorname{Sing}(X))=0$.

We can use the previous lemma to classify those varieties for which the equality in Problem (1.4) is achieved. Let us first recall the following definitions. 
(1.5.1) Definitions ([Z, p. 15]). Let $X \subset \mathbb{P}^{N}$ be a projective variety and $Y \subset X$ a connected subvariety of $X$. Let $\Delta_{Y}=\{(y, y): y \in Y\}$ be the relative diagonal of $Y$ in $X$. We define the variety of secants to $X$ relative to $Y$ as $S(Y, X)=$ $\overline{\bigcup_{(x, y) \in X \times Y \backslash \Delta_{Y}}\langle x, y\rangle}$, where $\langle x, y\rangle$ is the line joining $x$ and $y$. If $Y \cap \operatorname{Sing}(X)=\emptyset$ we define the variety of tangents to $X$ relative to $Y$ as $T(Y, X)=\overline{\bigcup_{y \in Y} T_{X, y}}$, where $T_{X, y}$ is the embedded projective tangent space to $X$ at $y \in Y$.

Problem (1.4) is settled as follows.

(1.6) Theorem. Let $X \subset \mathbb{P}^{N}$ be a non-degenerate projective variety, such that $\operatorname{dim}\left(X^{\vee}\right)=n-b-1(b=\operatorname{dim}(\operatorname{Sing}(X)))$. If $b=-1$, then $X \subset \mathbb{P}^{N}$ is either an Ein variety (see Example (1.3.1)(i)) or a smooth hypersurface. If $b \geq 0$, then $X \subset \mathbb{P}^{N}$ is a cone with its vertex a linear space of dimension $b$ over a smooth variety $Y \subset \mathbb{P}^{N-1-b}$ which is either an Ein variety or a smooth hypersurface.

Proof. If $b=-1$, that is, if $\operatorname{Sing}(X)=\emptyset$, we have that $X \subset \mathbb{P}^{N}$ is either a smooth hypersurface or an Ein variety. Then we can suppose $b \geq 0$. Argue by induction on $b$. Take $b=0$, that is, $\operatorname{dim}\left(X^{\vee}\right)=n-1$. If $X \subset \mathbb{P}^{N}$ is not a cone, then, by Lemma (1.5), the general contact locus $R$ avoids the singular points of $X$. By the Terracini Lemma [Z, Th. 1.4] either $\operatorname{dim}(S(R, X))=N+1$, contradicting $S(R, X) \subseteq \mathbb{P}^{N}$, or $T(R, X)=S(R, X)$. But there exists a hyperplane containing $T(R, X)$, contradicting the fact that $X \subset \mathbb{P}^{N}$ is non-degenerate. This means that $X \subset \mathbb{P}^{N}$ is a cone whose vertex is a point. In particular there is only one singular point. If $X \subset \mathbb{P}^{N}$ is a cone with vertex $p$, then a general hyperplane section is a non-singular variety $Y \subset \mathbb{P}^{N-1}$ for which $\operatorname{dim}\left(Y^{\vee}\right)=\operatorname{dim}(Y)$. This means that $Y \subset \mathbb{P}^{N-1}$ is either an Ein variety or a hypersurface. Now take $b>0$. For a general hyperplane section $Y \subset \mathbb{P}^{N-1}$ it holds that $\operatorname{dim}(\operatorname{Sing}(Y))=b-1$ and $\operatorname{dim}\left(Y^{\vee}\right)=\operatorname{dim}(Y)-b-1\left(\right.$ since $\left.\operatorname{dim}\left(Y^{\vee}\right)=\operatorname{dim}\left(X^{\vee}\right)\right)$. By induction $Y \subset \mathbb{P}^{N-1}$ is a cone whose vertex is $\mathbb{P}^{b-1}$. This means that the singular locus of $X$ is linear (its general hyperplane section is linear) and that $X \subset \mathbb{P}^{N}$ is a cone whose vertex is a $b$-plane.

Now we can come back to Problem (1.3). Our previous results has the following consequence.

(1.7) Corollary. For any triplet $(X, L, V)$ as in Problem (1.3), $\phi_{V}(X) \subset \mathbb{P}^{N}$ is one of the following: a smooth hypersurface, an Ein variety or a cone.

The following lemma, useful for our classification purposes, fits into the general perspective of studying the discriminant locus $\mathcal{D}(X, V)$ looking at $\phi_{V}(X) \subset \mathbb{P}^{N}$.

(1.8) Cone Lemma. Let $(X, L, V)$ be a triplet as in Section (0.0). If $\phi_{V}(X) \subset \mathbb{P}^{N}$ is a not linear cone of vertex $T=\mathbb{P}^{b}$ over a smooth variety $Y \subset \mathbb{P}^{N-1-b}$, then $\mathcal{J}_{n}(X, V) \neq \emptyset$ and $b=0$.

Proof. Let us recall [LPS1, (1.3.1)] that $x \in \mathcal{J}_{n}(X, V)$ if and only if $|V-x|=$ $|V-2 x|$. We proceed by induction on the dimension of the vertex of the cone.

Suppose $b=0$. Let $p \in \phi_{V}(X)$ be the vertex of the cone and let $p_{0} \in \phi_{V}^{-1}(p)$. We are going to show that any divisor in $\left|V-p_{0}\right|$ is singular at $p_{0}$. Consider $D \in\left|V-p_{0}\right|$. Note that $\operatorname{dim}\left(\left|V-p_{0}\right|\right)=\operatorname{dim}(|V|)-1 \geq n$. Hence, there exist $n$ points on $X$, say $p_{1}, \ldots, p_{n}$, such that $D \in\left|V-p_{0}-p_{1}-p_{2}-\cdots-p_{n}\right|$ and the lines $T_{i}=\left\langle p, \phi_{V}\left(p_{i}\right)\right\rangle \subset \phi_{V}(X)$ satisfy the condition $\operatorname{dim}\left(\left\langle T_{1}, \ldots, T_{n}\right\rangle\right)=n$. 
Otherwise the general hyperplane section of $\phi_{V}(X)$ through $p$ cuts $Y$ in a degenerate variety, contradicting the non-linearity of $\phi_{V}(X)$. Take $D_{i}=\phi_{V}^{-1}\left(T_{i}\right) \subset D$. Since $\operatorname{dim}\left(\left\langle T_{1}, \ldots, T_{n}\right\rangle\right)=n$, we can produce $n$ analytic $\operatorname{arcs} \alpha_{i}(t) \subset D_{i}$ such that $\alpha_{i}(0)=$ $p_{0}$ and the vectors $\alpha_{1}^{\prime}(0), \ldots, \alpha_{n}^{\prime}(0)$ are linearly independent. Thus the Zariski tangent space to $D$ at $p_{0}$ has dimension bigger than or equal to $n$, and we conclude that $D$ is singular at $p_{0}$.

Suppose $b>0$. Let $p \in T\left(=\mathbb{P}^{b}\right) \subset \phi_{V}(X)$ be a point in the vertex of the cone and let $p_{0} \in \phi_{V}^{-1}(p)$. We are going to show that any divisor in $\left|V-p_{0}\right|$ is singular at $p_{0}$. Let $D$ and $D^{\prime}$ be two general elements in $\left|V-p_{0}\right|$ and suppose, by contradiction, that they are smooth at $p_{0}$. By induction $D \cap D^{\prime}$ is singular at $p_{0}$. This means that the Zariski tangent space to $D$ at $p_{0}$ is equal to that of $D^{\prime}$ at $p_{0}$ (as vector subspaces of the Zariski tangent space to $X$ at $p_{0}$ ), that is, they are not transversal. In a similar way to the construction in the case $b=0$ it is possible to choose two sets of points of $X$, say $p_{1}, \ldots, p_{n-1}$ and $q_{1}, \ldots q_{n-1}$, such that:

i) $D \in\left|V-p_{0}-\cdots-p_{n-1}\right|$ and $D^{\prime} \in\left|V-p_{0}-q_{1}-\cdots-q_{n-1}\right|$,

ii) $H_{p_{1}, \ldots, p_{n-b}}=\left\langle\left\langle p, \phi_{V}\left(p_{1}\right)\right\rangle, \ldots,\left\langle p, \phi_{V}\left(p_{n-b}\right)\right\rangle\right\rangle=\mathbb{P}^{n-b}$

iii) $H_{q_{1}, \ldots, q_{n-b}}=\left\langle\left\langle p, \phi_{V}\left(q_{1}\right)\right\rangle, \ldots,\left\langle p, \phi_{V}\left(q_{n-b}\right)\right\rangle\right\rangle=\mathbb{P}^{n-b}$,

iv) $H_{p_{1}, \ldots, p_{n-b}} \cap T=H_{q_{1}, \ldots, q_{n-b}} \cap T=\{p\}$,

$\mathrm{v})$ there exist $p_{n-b+1}^{\prime}, \ldots, p_{n-1}^{\prime}\left(q_{n-b+1}^{\prime}, \ldots, q_{n-1}^{\prime}\right.$, respectively) in $T$ such that the lines $\left\langle\phi_{V}\left(p_{j}\right), p_{j}^{\prime}\right\rangle\left(\left\langle\phi_{V}\left(q_{j}\right), q_{j}^{\prime}\right\rangle\right.$, respectively) are contained in $\phi_{V}(D)$ (in $\phi_{V}\left(D^{\prime}\right)$, respectively).

Actually, ii), iii) and iv) are a consequence of the fact that $\phi_{V}(X)$ is a not linear cone over $Y$ and then the hyperplane sections of $\phi_{V}(X)$ corresponding to $D$ and $D^{\prime}$ contain a cone, say $C$ and $C^{\prime}$, of vertex $p$ over a hyperplane section of $Y$. For v) we have to observe that for a general point $\phi_{V}(z) \in \phi_{V}(D) \backslash C\left(\phi_{V}\left(z^{\prime}\right) \in \phi_{V}\left(D^{\prime}\right) \backslash C^{\prime}\right.$, respectively) there exists a line in the hyperplane section of $\phi_{V}(X)$ corresponding to $D$ ( $D^{\prime}$, respectively) cutting on $T$ a point different from $p$.

Finally, define the lines $T_{i}=\left\langle p, \phi_{V}\left(p_{i}\right)\right\rangle\left(T_{i}^{\prime}=\left\langle p, \phi_{V}\left(q_{i}\right)\right\rangle\right.$, respectively) for $i=1, \ldots, n-b$ and $T_{j}=\left\langle p, p_{j}^{\prime}\right\rangle\left(T_{j}=\left\langle p, q_{j}^{\prime}\right\rangle\right.$, respectively) for $j=n-b+1, \ldots, n-1$. This produces two sets of analytic arcs, say $\left\{\alpha_{i}(t)\right\}$ and $\left\{\beta_{i}(t)\right\}$ for $i=1, \ldots, n-1$, such that $\alpha_{i}(0)=\beta_{i}(0)=p_{0}$ and $\alpha_{i}(t) \in D, \beta_{i}(t) \in D^{\prime}$ for any $i=1, \ldots, n-1$. But this gives a contradiction, because the vector space spanned by $\alpha_{1}^{\prime}(0), \ldots, \alpha_{n-1}^{\prime}(0)$ is different from that spanned by $\beta_{1}^{\prime}(0), \ldots, \beta_{n-1}^{\prime}(0)$.

This implies that $\mathcal{J}_{n}(X, V) \supseteq \phi_{V}^{-1}(T)$, hence $\operatorname{dim}\left(\mathcal{J}_{n}(X, V)\right) \geq b$. But then $b=0$, by [LPS1, Th. 1.2].

(1.9) Lemma. Let $(X, L, V)$ be a triplet as in Section (0.0). If $\phi_{V}(X) \subset \mathbb{P}^{N}$ is a smooth variety and $\operatorname{dim}(\mathcal{D}(X, V))=\operatorname{dim}\left(\mathcal{D}_{0}(X, V)\right)<N-1$, then $\phi_{V}\left(\mathcal{J}_{1}(X, V)\right) \cap$ $R=\emptyset$, where $R$ stands for the contact locus of $\phi_{V}(X) \subset \mathbb{P}^{N}$ with a general $H \in \phi_{V}(X)^{\vee}$. In particular, if $\mathcal{J}_{1}(X, V) \neq \emptyset$, then the Picard group of $\phi_{V}(X)$, $\operatorname{Pic}\left(\phi_{V}(X)\right)$, is not isomorphic to $\mathbb{Z}$.

Proof. If $\mathcal{J}_{1}(X, V)=\emptyset$, then the first assertion is obvious. If $\mathcal{J}_{1}(X, V) \neq \emptyset$, since $\phi_{V}(X)$ is smooth and not isomorphic to $X$, then $\mathcal{J}_{1}(X, V) \in \operatorname{Pic}(X) \backslash\{0\}$. Consider $H \in \phi_{V}(X)^{\vee}$ general; then $R:=\operatorname{Sing}\left(H \cap \phi_{V}(X)\right)=\mathbb{P} \operatorname{def}(X, V)$ with $\operatorname{def}(X, V)>0$. Let us suppose $R \cap B \neq \emptyset$, where $B \subset \mathbb{P}^{N}$ stands for an irreducible component of $\phi_{V}\left(\mathcal{J}_{1}(X, V)\right)$. Then

(1.9.1) there exists $b \in B$ such that $T_{\phi_{V}(X), b} \subset H$. 
Recall the following definitions of the conormal varieties: $\mathcal{X}_{\phi_{V}(X)}$ (respectively $\left.\mathcal{X}_{B}\right)$ is the set $\left\{(x, H): x \in \phi_{V}(X), T_{\phi_{V}(X), x} \subset H\right\} \subset \mathbb{P}^{N} \times \mathbb{P}^{N \vee}$ (respectively the closure of the set $\left.\left\{(b, H): b \in B \backslash \operatorname{Sing}(B), T_{B, b} \subset H\right\} \subset \mathbb{P}^{N} \times \mathbb{P}^{N \vee}\right)$. Consider the set $\mathcal{X}^{0}=\left\{(b, H): b \in B \backslash \operatorname{Sing}(B), T_{\phi_{V}(X), b} \subset H\right\} \subset \mathbb{P}^{N} \times \mathbb{P}^{N \vee}$. Since $T_{B, b} \subset T_{\phi_{V}(X), b}$ for any $b \in B \backslash \operatorname{Sing}(B)$ it holds that $\mathcal{X}^{0} \subset \mathcal{X}_{B} \cap \mathcal{X}_{\phi_{V}(X)}$. Hence, in particular, $\overline{\mathcal{X}^{0}} \subset \mathcal{X}_{B}$. This means that any hyperplane tangent to $\phi_{V}(X)$ in a point of $B$ is contained in $B^{\vee}$, in particular, by (1.9.1), the general $H \in$ $\phi_{V}(X)^{\vee}$ is an element of $B^{\vee}$ and then $\phi_{V}(X)^{\vee} \subset B^{\vee}$. Since $B^{\vee} \subset \mathcal{D}(X, V)$ then $\operatorname{dim}\left(B^{\vee}\right) \leq \operatorname{dim}(\mathcal{D}(X, L))=\operatorname{dim}\left(\phi_{V}(X)^{\vee}\right)$ and so $\phi_{V}(X)^{\vee}=B^{\vee}$. By biduality it means $\phi_{V}(X)=B$, a contradiction.

If $\operatorname{Pic}\left(\phi_{V}(X)\right) \cong \mathbb{Z}$, then it is generated by $\mathcal{L}:=\left.\mathcal{O}_{\mathbb{P}^{N}}(1)\right|_{\phi_{V}(X)}$. Since $\phi_{V}$ is not an isomorphism, then either $\mathcal{J}_{1}(X, V)=\emptyset$ or $B$ is linearly equivalent to $m \mathcal{L}$ with $m \geq 1$. Hence $\operatorname{Sing}\left(H \cap \phi_{V}(X)\right) \cap B \neq \emptyset$, contradicting the first part of the lemma.

(1.10) Theorem. For $(X, L, V)$ as in Problem (1.3) one of the following holds:

(i) $\phi_{V}(X)$ is a smooth hypersurface;

(ii) $L$ is very ample, $\phi_{V}$ an isomorphism, and $\phi_{V}(X) \subset \mathbb{P}^{N}$ is an Ein variety;

(iii) $\phi_{V}(X) \subset \mathbb{P}^{N}$ is the Segre embedding of $\mathbb{P}^{1} \times \mathbb{P}^{n-1}$ in $\mathbb{P}^{2 n-1}, n \geq 3$.

Proof. If $\phi_{V}(X)$ is a cone, then $\mathcal{J}_{n}(X, V) \neq \emptyset$ by the Cone Lemma. Hence $\operatorname{dim}(\mathcal{D})=\operatorname{dim}(|V|)-1$. This means: $\operatorname{dim}(|V|)-1=\operatorname{dim}(X)-1-\operatorname{dim}\left(\operatorname{Sing}\left(\phi_{V}(X)\right)\right)$, a contradiction because the singular locus of $\phi_{V}(X)$ is non-empty and $\phi_{V}(X)$ is not linear. Hence, by Corollary (1.7), $\phi_{V}(X) \subset \mathbb{P}^{N}$ is either a smooth hypersurface or an Ein variety. Then either (i) or (ii) holds or $\phi_{V}(X) \subset \mathbb{P}^{N}$ is an Ein variety and $\phi_{V}$ is not an isomorphism. In the latter case, if $n>2 N / 3$, then the Barth-Larsen Theorem combined with Lemma (1.9) leads to a contradiction. Thus $n \leq 2 N / 3$ and so $\phi_{V}(X)$ is the Segre embedding of $\mathbb{P}^{1} \times \mathbb{P}^{n-1}$ in $\mathbb{P}^{2 n-1}$ due to Ein classification [E1, Th. 4.5].

(1.10.1) It is clear that (i) and (ii) are effective. We can give a description of (iii). Suppose $\phi_{V}(X)=\mathbb{P}^{1} \times \mathbb{P}^{n-1} \subset \mathbb{P}^{2 n-1}$. Let us call $\pi_{1}: \mathbb{P}^{1} \times \mathbb{P}^{n-1} \rightarrow \mathbb{P}^{1}$ the first projection. By Lemma (1.9) any component $B$ of $\phi_{V}\left(\mathcal{J}_{1}(X, V)\right)$ does not meet the general contact locus of $\phi_{V}(X)$. Then $B$ is contained in a union of fibres of $\pi_{1}$. Now consider the map: $\pi_{1} \circ \phi_{V}: X \rightarrow \mathbb{P}^{1}$. Its general fibre $F:=\left(\pi_{1} \circ \phi_{V}\right)^{-1}(p), p \in \mathbb{P}^{1}$, is smooth, equidimensional, and $\left.\phi_{V}\right|_{F}: F \rightarrow \pi_{1}^{-1}(p)=\mathbb{P}^{n-1}$ does not ramify. Then $F$ is a disjoint union of linear spaces $R$ of dimension $n-1$. Let us remark that the differential of the map $\phi_{V}$ is giving an isomorphism between $\left.T_{X}\right|_{R}$, the tangent bundle to $X$ restricted to $R$, and $\left.T_{\phi_{V}(X)}\right|_{\phi_{V}(R)}$. Then the normal bundle $\mathcal{N}_{R / X}$ is isomorphic to $\mathcal{N}_{\phi_{V}(R) / \phi_{V}(X)}$ and hence trivial. By adjunction formula $\left.K_{X}\right|_{R}=K_{R}=\mathcal{O}_{R}(-n)$. Hence $\left.\left(K_{X}+(n-1) L\right)\right|_{R}=\mathcal{O}_{R}(-1)$. Then the nef value of $(X, L)$ is bigger than $\operatorname{dim}(X)-1$, and so we have by [I, Th. 1.5] and [BS, Th. 11.1.1] that $(X, L)$ is one of the following: a scroll over a smooth irreducible curve $C, p: X \rightarrow C$, a quadric $\left(Q^{n}, \mathcal{O}_{Q^{n}}(1)\right)$, or the Veronese surface. Only the first possibility occurs. Then we can define $g: C \rightarrow \mathbb{P}^{1}$ to obtain a commutative diagram such as the following:

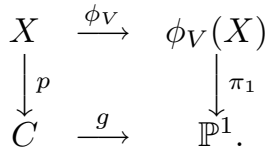


In fact this shows that for any $(X, L, V)$ as in Theorem $(1.10)$, if $\phi_{V}(X)=\mathbb{P}^{1} \times$ $\mathbb{P}^{n-1} \subset \mathbb{P}^{2 n-1}, n \geq 2$, then there exists a smooth irreducible curve $C$ and a map $g: C \rightarrow \mathbb{P}^{1}$ such that $X=\mathbb{P}_{C}\left(g^{*}\left(\mathcal{O}_{\mathbb{P}^{1}}(1)^{\oplus n}\right)\right)=C \times \mathbb{P}^{n-1}$ and $\phi_{V}$ is induced by $g$.

(1.11) Corollary. For $(X, L, V)$ as in Problem (1.3), if $\operatorname{def}(X, V)>0$, then $(X, L)$ is swept out by $\mathbb{P}^{\operatorname{def}(X, V)}$ 's. That is, in this particular case, [PS1, Conj. 2.11] holds.

(1.12) Let us make some comments about the next case, that is, triplets $(X, L, V)$ as in Section $(0.0), \phi_{V}(X)$ not linear and $\operatorname{dim}(\mathcal{D}(X, V))=n-\operatorname{dim}\left(\operatorname{Sing}\left(\phi_{V}(X)\right)\right)$. In this case, combining (1.1) with the inequality $\operatorname{dim}\left(\mathcal{D}_{0}\right) \leq \operatorname{dim}(\mathcal{D}), \phi_{V}(X) \subset \mathbb{P}^{N}$ verifies either

i) $\operatorname{dim}\left(\phi_{V}(X)^{\vee}\right)=\operatorname{dim}\left(\phi_{V}(X)\right)-1-\operatorname{dim}\left(\operatorname{Sing}\left(\phi_{V}(X)\right)\right)$, or

ii) $\operatorname{dim}\left(\phi_{V}(X)^{\vee}\right)=\operatorname{dim}\left(\phi_{V}(X)\right)-\operatorname{dim}\left(\operatorname{Sing}\left(\phi_{V}(X)\right)\right)$.

If i) holds, by Theorem (1.6), $\phi_{V}(X)$ is either a smooth variety or a cone. In the former case, $\phi_{V}(X) \subset \mathbb{P}^{N}$ is either a smooth hypersurface or an Ein variety. If $\phi_{V}(X) \subset \mathbb{P}^{N}$ is a smooth hypersurface, then $\operatorname{dim}(\mathcal{D}(X, V))=N$, a contradiction. If $\phi_{V}(X) \subset \mathbb{P}^{N}$ is an Ein variety we have examples: just take $\pi_{k}: Y \rightarrow \mathbb{P}^{2}$ a smooth ramified cyclic covering of the plane (of degree $k$ ) and consider the Segre embedding of the image of $I d \times \pi_{k}: \mathbb{P}^{1} \times Y \rightarrow \mathbb{P}^{1} \times \mathbb{P}^{2}$. Then $\mathcal{D}_{0}=\left(\mathbb{P}^{1} \times \mathbb{P}^{2}\right)^{\vee}$ and $\mathcal{D}_{1}$ is the dual variety of a smooth ruled surface of $\mathbb{P}^{5}$, that is, a hypersurface of $\left(\mathbb{P}^{5}\right)^{\vee}$. This means $\operatorname{dim}(\mathcal{D})=4=\operatorname{dim}\left(\phi_{V}(X)\right)+1$. If $\phi_{V}(X) \subset \mathbb{P}^{N}$ is a cone, then $\mathcal{J}_{n}(X, V) \neq \emptyset$ by the Cone Lemma. Hence $N-1=\operatorname{dim}(\mathcal{D}(X, V))=n-\operatorname{dim}\left(\operatorname{Sing}\left(\phi_{V}(X)\right)\right), n=N-1$, and $\operatorname{dim}\left(\operatorname{Sing}\left(\phi_{V}(X)\right)\right)=0$. Example (0.2) shows that this possibility is effective.

In case ii) suppose that $\phi_{V}(X) \subset \mathbb{P}^{N}$ is smooth. Then

$$
\operatorname{dim}\left(\phi_{V}(X)\right)^{\vee}=\operatorname{dim}\left(\phi_{V}(X)\right)+1=\operatorname{dim} \mathcal{D}(X, V) .
$$

By Lemma (1.9) we get the following possibilities:

ii.1) $\phi_{V}(X) \subset \mathbb{P}^{N}$ is a smooth codimension 2 variety whose dual variety is a hypersurface;

ii.2) $\phi_{V}$ is an isomorphism and $\phi_{V}(X) \subset \mathbb{P}^{N}$ is a positive defect variety for which $\operatorname{dim}\left(\phi_{V}(X)^{\vee}\right)=\operatorname{dim}\left(\phi_{V}(X)\right)+1$;

ii.3) in the remaining cases $n \leq 2 N / 3$ by the Barth-Larsen Theorem. Then we can use the classification of [M1, Th. 3.1] to assure that $\phi_{V}(X) \subset \mathbb{P}^{N}$ is a scroll over a smooth curve $C, \pi: \phi_{V}(X) \rightarrow C$, embedded in $\mathbb{P}^{2 n}$. Let us show examples. Take $C^{\prime}$ as a rational or elliptic smooth curve and $\mathcal{E}^{\prime}$ as a very ample vector bundle of rank $n$ on $C^{\prime}$ such that $Y=\mathbb{P}\left(\mathcal{E}^{\prime}\right)$ can be embedded in $\mathbb{P}^{2 n}$ by a $(2 n+1)$-dimensional subspace $V^{\prime} \subseteq H^{0}\left(Y, h^{\prime}\right)$, where $h^{\prime}$ stands for the tautological line bundle. Let $f: C \rightarrow C^{\prime}$ be a finite morphism, let $\mathcal{E}=f^{*}\left(\mathcal{E}^{\prime}\right)$, and set $X=\mathbb{P}(\mathcal{E})$. Then we have a finite morphism $F: X \rightarrow Y$ induced by $f$, and taking $V=F^{*}\left(V^{\prime}\right)$ we get an example of this situation. These are essentially the only examples by arguments similar to that of Section (1.10.1).

When $\phi_{V}(X)$ is singular, the situation changes with respect to the extremal case. In fact, the Terracini Lemma cannot be used to assure that $\phi_{V}(X) \subset \mathbb{P}^{N}$ is a cone. We refer to Example (3.2) for an example where $\phi_{V}(X) \subset \mathbb{P}^{3}$ is a surface that is not a cone, $\operatorname{dim}\left(\operatorname{Sing}\left(\phi_{V}(X)\right)\right)=1$, and the dual variety is a curve.

We conclude the section with the following lemma that opens the possibility of classifying positive defect triplets $(X, L, V)$ as in Section (0.0) by an inductive process on the dimension of $X$. 
(1.13) Lemma. Let $(X, L, V)$ be as in Section (0.0). Take $X_{1} \in|V|$ general, $L_{1}=$ $\left.L\right|_{X_{1}}$ and $V_{1}$ the image of $V$ by the restriction map $r: H^{0}(X, L) \rightarrow H^{0}\left(X_{1}, L_{1}\right)$. Then $\operatorname{def}\left(X_{1}, V_{1}\right) \geq \operatorname{def}(X, V)-1$.

Proof. The proof comes from the characterization of the defect in terms of the vanishing of some Chern classes of the first jet bundle [LPS1, (2.7)]. Consider the following exact sequence:

$$
0 \rightarrow T_{X_{1}}^{*}\left(L_{1}\right) \rightarrow J_{1}\left(L_{1}\right) \rightarrow L_{1} \rightarrow 0 .
$$

The Chern polynomial of $J_{1}\left(L_{1}\right)$ can be expressed as the product of the Chern polynomials of $T_{X_{1}}^{*}\left(L_{1}\right)$ and $L_{1}$. Now, taking into account the exact sequences

$$
\left.0 \rightarrow \mathcal{O}_{X_{1}} \rightarrow T_{X}^{*}\left(L_{1}\right)\right|_{X_{1}} \rightarrow T_{X_{1}}^{*}\left(L_{1}\right) \rightarrow 0
$$

and

$$
\left.\left.0 \rightarrow T_{X}^{*}(L)\right|_{X_{1}} \rightarrow J_{1}(L)\right|_{X_{1}} \rightarrow L_{1} \rightarrow 0,
$$

we see that the Chern polynomial of $J_{1}\left(L_{1}\right)$ is exactly the same as that of $\left.J_{1}(L)\right|_{X_{1}}$. By [LPS1, (2.7)], if $\operatorname{def}(X, V)=k>0$, then $c_{\operatorname{dim}(X)-i+1}\left(J_{1}(L)\right)=0$ for all $i \leq k$. This means that $c_{\operatorname{dim}\left(X_{1}\right)-i+2}\left(J_{1}\left(L_{1}\right)\right)=0$ for all $i \leq k$. Hence $\operatorname{def}\left(X_{1}, V_{1}\right) \geq k-1$, once again by [LPS1, (2.7)].

\section{CyClic Coverings}

Let $(X, L, V)$ be a triplet as in Section (0.0). Our general goal is to study triplets $(X, L, V)$ for which the discriminant locus has small dimension. Since $\operatorname{def}(X, V)>0$ implies that the classical defect of $\phi_{V}(X) \subset \mathbb{P}^{N}$ is bigger than 0 , it seems a first step to consider situations for which $\phi_{V}(X) \subset \mathbb{P}^{N}$ is a smooth positive defect variety. In this context it is natural to consider the following setting which is slightly more general than that in LPS2, Ex. 4.1.3].

(2.1) Let $X, Y$ be smooth complex projective varieties of dimension $n \geq 2$, and let $\pi: X \rightarrow Y$ be a branched cyclic cover of degree $m \geq 2$. Let $\mathcal{L}$ be a very ample line bundle on $Y$ and $\mathcal{V} \subseteq H^{0}(Y, \mathcal{L})$ a vector subspace (of dimension $N+1$ ) of global sections defining an embedding $\phi_{\mathcal{V}}: Y \rightarrow \mathbb{P}^{N}$. Set $L:=\pi^{*} \mathcal{L}, V=\pi^{*} \mathcal{V}$. We assume that

$$
\operatorname{def}(X, V)>0 .
$$

A conjecture stated in [LPS1, (2.11)] says that for a triplet $(X, L, V)$ as in Section $(0.0)$, if $\delta=\operatorname{def}(X, V)>0$, then $(X, L)$ is covered by linear $\mathbb{P}^{\delta} \delta$ s. The analog of this conjecture with def $_{0}$ instead of def has been proved in LPS2 under the extra assumption that $\phi_{V}$ is generically one-to-one. Note that the def $_{0}$ version of the conjecture is false without this further assumption on $\phi_{V}$, as shown by Example (0.2). Here we prove a structure theorem for positive defect varieties as in Section (2.1), in connection with the def version of the conjecture and in the spirit of the structure theorem of [BFS, Th. 1.2] for classical positive defect varieties.

Let $B$ be the branch locus of $\pi$. Then $B$ is a smooth divisor, $X$ being smooth. So there are no jumping sets $\mathcal{J}_{i}(X, V)$ with $i \geq 2$, while $\mathcal{J}_{1}(X, V)$ is the ramification locus of $\pi$. Hence there is only one extra part $\mathcal{D}_{1}$ in $\mathcal{D}(X, V)$, coming from the irreducible components of the ramification locus of $\pi$, which is isomorphic to $B$. Now look at the embedding $\phi_{\mathcal{V}}: Y \rightarrow \mathbb{P}^{N}$. Then $\mathcal{D}(X, V)=\mathcal{D}_{0} \cup \mathcal{D}_{11} \cup \cdots \cup \mathcal{D}_{1 l}$, where $\mathcal{D}_{0}$ can be identified with the dual variety $\phi_{\mathcal{V}}(Y)^{\vee}$ and $\mathcal{D}_{1 j}$ can be identified with the dual variety in $|V|=\mathbb{P}^{N \vee}$ of the $j$-th irreducible component of $\phi_{\mathcal{V}}(B)$, 
$j=1, \ldots, l$. So, recalling (0.0.1), condition (2.1.0) implies both $\operatorname{def}_{0}(X, V)=$ $\operatorname{def}(Y, \mathcal{V})>0$ and $\operatorname{def}_{1}(X, V)>0$. The former inequality says that $\phi_{\mathcal{V}}(Y)$ must have positive defect. On the other hand $\operatorname{def}_{1}(X, V)$ is the minimum of the classical defects of the irreducible components of $\phi_{\mathcal{V}}(B) \subset \mathbb{P}^{N}$. Hence the latter inequality tells us that every irreducible component of $\phi_{\mathcal{V}}(B)$ must have positive defect. In conclusion, we get

$$
0<\operatorname{def}(X, V)=\min \left\{\operatorname{def}\left(\phi_{\mathcal{V}}(Y)\right), \operatorname{def}\left(B_{1}^{\prime}\right)\right\},
$$

where $B_{1}^{\prime}$ is the irreducible component of $\phi_{\mathcal{V}}(B) \subset \mathbb{P}^{N}$ attaining the minimum defect. In particular this has the following implication.

(2.2) Proposition. Let $\pi: X \rightarrow Y$ be a branched cyclic cover of degree $m \geq 2$, let $\mathcal{L}, L, V$ be as in Section (2.1), and let $B$ be the branch divisor on $Y$. If $B$ is irreducible and the linear system $|B-\mathcal{L}|$ defines a generically one-to-one map, then $\operatorname{def}(X, V)=0$.

Proof. By contradiction, suppose that (2.1.0) holds. Then $\phi_{\mathcal{V}}(B)$ has positive defect by what we said before. But this circumstance cannot occur, in view of the following lemma.

(2.3) Lemma. Let $\mathcal{L}$ be a very ample line bundle on a smooth projective variety $Y$, and let $B$ be a smooth irreducible hypersurface of $Y$. If the linear system $|B-\mathcal{L}|$ defines a generically one-to-one map, then $\operatorname{def}\left(B,\left.\mathcal{L}\right|_{B}\right)=0$.

Proof. The proof follows that of Theorem 1.3, Part (b) in [E2, pp. 898-899], by replacing the line bundle $\mathcal{O}_{X}(d-1)$ appearing there with our $B-\mathcal{L}$.

Let us continue our discussion assuming (2.1.0). Of course $\phi_{\mathcal{V}}(Y) \subset \mathbb{P}^{N}$ is non-degenerate and, according to what we said, it has positive defect. Therefore $(Y, \mathcal{L})$ is covered by lines. Moreover there is an irreducible component $\mathcal{H}$ of the corresponding Hilbert scheme, whose lines cover $Y$. Let $\ell \subset Y$ be any line of $\mathcal{H}$. Then $\ell \mathcal{L}=1$ and $\ell B \geq 0$, since $B$ is a hypersurface of $Y$, while the $\ell$ 's cover $Y$. In fact we have

(2.4) Proposition. $\ell B=0$ for any $\ell \in \mathcal{H}$.

Proof. First recall that $B \in|m A|$ for some line bundle $A \in \operatorname{Pic}(Y)$, since $\pi$ is a cyclic cover of degree $m$. So, if $\ell$ is any line of $Y$ such that $\ell B>0$, then we have

$$
\ell B=m \ell A \geq m \geq 2 .
$$

Let $B_{0}^{\prime}$ be any irreducible component of $\phi_{\mathcal{V}}(B)$. Let $b_{0}^{\prime} \in B_{0}^{\prime}$ be a general point and $H$ a general tangent hyperplane to $B_{0}^{\prime}$ at $b_{0}^{\prime}$. Then $T_{B_{0}^{\prime}, b_{0}^{\prime}} \subset H$, but $H$ does not contain $T_{\phi_{\mathcal{V}}(Y), b_{0}^{\prime}}$. Since $B_{0}^{\prime}$ is a positive defect variety there exists a line $\ell_{0}$ through $b_{0}^{\prime}$ in the contact locus of $H$ and $B_{0}^{\prime}$. Consider the conormal bundle sequence of $B_{0}^{\prime} \subset \phi_{\mathcal{V}}(Y) \subset \mathbb{P}^{N}$ twisted by $\mathcal{L}$ and restricted to $\ell_{0}$ :

$$
\left.\left.0 \rightarrow \mathcal{N}_{\phi_{\mathcal{V}}(Y)}^{*}(\mathcal{L})\right|_{\ell_{0}} \rightarrow \mathcal{N}_{B_{0}^{\prime}}^{*}(\mathcal{L})\right|_{\ell_{0}} \rightarrow \mathcal{O}_{\ell_{0}}\left(\mathcal{L}-B_{0}^{\prime}\right) \rightarrow 0
$$

We claim that

$$
\ell_{0} B_{0}^{\prime} \leq 1
$$

Since $\ell_{0} \mathcal{L}=1$, if $B_{0}^{\prime} \ell_{0}>1$, then $\mathcal{O}_{\ell_{0}}\left(\mathcal{L}-B_{0}^{\prime}\right)=\mathcal{O}_{\mathbb{P}^{1}}(a)$ for some integer $a<0$. This gives $h^{0}\left(\left.\mathcal{N}_{\phi_{\mathcal{V}}(Y)}^{*}(\mathcal{L})\right|_{\ell_{0}}\right)=h^{0}\left(\left.\mathcal{N}_{B_{0}^{\prime}}^{*}(\mathcal{L})\right|_{\ell_{0}}\right)$. By [M2, Prop. 3.6, i) and ii $)$ this means that $\left\langle T\left(\ell_{0}, \phi_{\mathcal{V}}(Y)\right)\right\rangle=\left\langle T\left(\ell_{0}, B_{0}^{\prime}\right)\right\rangle$. Note that $H$ contains $T\left(\ell_{0}, B_{0}^{\prime}\right)$ since $\ell_{0}$ 
is in the contact locus, hence it contains the linear span $\left\langle T\left(\ell_{0}, B_{0}^{\prime}\right)\right\rangle$. Then, due to the above equality, $H$ would also contain $T\left(\ell_{0}, \phi_{\mathcal{V}}(Y)\right)$. But this is impossible since, according to our choice, $H \nsupseteq T_{\phi_{\mathcal{V}}(Y), b_{0}^{\prime}}$. This shows (2.4.1). Now, if $\phi_{\mathcal{V}}(B)$ is irreducible this implies $\ell_{0} \phi_{\mathcal{V}}(B)=0$ by (2.4.0). On the other hand, if $\phi_{\mathcal{V}}(B)$ is reducible, then being smooth, its irreducible components do not meet each other. Hence $\ell_{0} \phi_{\mathcal{V}}(B)=\ell_{0} B_{0}^{\prime}$, because $\ell_{0}$ does not meet any other component of $\phi_{\mathcal{V}}(B)$. Thus, if $\ell_{0} \phi_{\mathcal{V}}(B)>0$ we get $\ell_{0} B_{0}^{\prime} \geq 2$ by (2.4.0) again, which gives a contradiction. Therefore $\ell_{0} \phi_{\mathcal{V}}(B)=0$ for any general line in a contact locus of any component of $\phi_{\mathcal{V}}(B)$. Now to prove the assertion it is enough to show that $\ell_{0}$ belongs to $\mathcal{H}$. So now we prove that there exists a hyperplane tangent to $\phi_{\mathcal{V}}(Y)$ along $l_{0}$. By [M2, Prop. 3.6, i) and ii $)]$ we have $\operatorname{dim}\left(\left\langle T\left(\ell_{0}, \phi_{\mathcal{V}}(Y)\right)\right\rangle\right)=N-h^{0}\left(\left.\mathcal{N}_{\phi_{\mathcal{V}}(Y)}^{*}(\mathcal{L})\right|_{\ell_{0}}\right)$. Hence, if

$$
h^{0}\left(\left.\mathcal{N}_{\phi_{\mathcal{V}}(Y)}^{*}(\mathcal{L})\right|_{\ell_{0}}\right)>0
$$

then there is a hyperplane containing $\left\langle T\left(\ell_{0}, \phi_{\mathcal{V}}(Y)\right)\right\rangle$. Let us prove (2.4.2). Denote by $k$ the defect of $B_{0}^{\prime}$; recall that $k>0$, by (2.1.1). Since $\operatorname{dim} B_{0}^{\prime}=n-1$, we get from [E1, Th. 2.3] that $\mathcal{N}_{\ell_{0} / B_{0}^{\prime}}=\mathcal{O}_{\mathbb{P}^{1}}(1)^{\oplus \frac{n+k-3}{2}} \oplus \mathcal{O}_{\mathbb{P}^{1}}^{\oplus \frac{n-1-k}{2}}$. Moreover, the normal bundle sequence of $\ell_{0} \subset B_{0}^{\prime} \subset \phi_{\mathcal{V}}(Y)$ is

$$
0 \rightarrow \mathcal{N}_{\ell_{0} / B_{0}^{\prime}} \rightarrow \mathcal{N}_{\ell_{0} / \phi_{\mathcal{V}}(Y)} \rightarrow \mathcal{O}_{\ell_{0}} \rightarrow 0
$$

because $\ell_{0} B_{0}^{\prime}=0$. Clearly this exact sequence splits, and then we have $\mathcal{N}_{\ell_{0} / \phi_{\mathcal{V}}(Y)}=$ $\mathcal{O}_{\mathbb{P}^{1}}(1)^{\oplus \frac{n+k-3}{2}} \oplus \mathcal{O}_{\mathbb{P}^{1}}^{\oplus \frac{n+1-k}{2}}$. Now, by dualizing the normal bundle sequence of $\ell_{0} \subset$ $\phi_{\mathcal{V}}(Y) \subset \mathbb{P}^{N}$ and twisting by $\mathcal{L}$ we get the exact sequence

$$
\left.0 \rightarrow \mathcal{N}_{\phi_{\mathcal{V}}(Y)}^{*}(\mathcal{L})\right|_{l_{0}} \rightarrow \mathcal{O}_{l_{0}}^{\oplus N-1} \rightarrow \mathcal{O}_{l_{0}}^{\oplus \frac{n+k-3}{2}} \oplus \mathcal{O}_{l_{0}}(\mathcal{L})^{\oplus \frac{n+1-k}{2}} \rightarrow 0 .
$$

Then $h^{0}\left(\left.\mathcal{N}_{\phi_{\mathcal{V}}(Y)}^{*}(\mathcal{L})\right|_{l_{0}}\right) \geq N-1-\frac{n+k-3}{2}-(n+1-k)$. So, to prove (2.4.2) it is enough to show that $N-1-\frac{n+k-3}{2}-(n+1-k)>0$. This is equivalent to $2 N-2-n-k+3-2 n-2+2 k=2 N-3 n+k-1>0$. Since $k>0$ it is enough to show that $2 N-3 n>0$. Suppose that $2 N-3 n \leq 0$; then $n \geq 2 N / 3$ and hence, by the Barth-Larsen Theorem, $\operatorname{Pic}\left(\phi_{\mathcal{V}}(Y)\right)$ is generated by the hyperplane section, but this contradicts the equality $\ell_{0} \phi_{\mathcal{V}}(B)=0$.

This has the following nice consequence.

(2.5) Proposition. Let $\pi: X \rightarrow Y$ be a branched cyclic cover of degree $m \geq 2$, let $\mathcal{L}, L, V$ be as in Section (2.1), and let $B$ be the branch divisor on $Y$. If either

(1) $Y$ has Picard number 1, or

(2) $B \in|d \mathcal{L}|$ for some integer $d \geq 1$,

then $\operatorname{def}(X, V)=0$.

Note that this happens, e.g., for the pair $(Y, \mathcal{L})=\left(\mathbb{P}^{n}, \mathcal{O}_{\mathbb{P}^{n}}(1)\right)$. As a consequence, for the pair $(X, L)$ in [LPS2, Ex. 4.1.3] we have that $\operatorname{def}(X, V)=0$.

Proof. In case (1), call $h$ the ample generator of $\operatorname{Pic}(Y)$. Then $B=b h$ for some integer $b \geq 1$. In particular $B$ is ample. In case $(2), B$ is very ample. If $\operatorname{def}(X, V)>$ 0 , by Proposition $(2.4)$, there is a line $\ell$ of $(Y, \mathcal{L})$ such that $\ell B=0$, contradicting the ampleness of $B$. 
Now we continue our discussion under the assumption (2.1.0). By Proposition (2.4) any contact locus of a hyperplane $H$ with $\phi_{\mathcal{V}}(Y)$ is either contained in $\phi_{\mathcal{V}}(B)$ or does not meet $\phi_{\mathcal{V}}(B)$. As in [BFS], let $\Phi: Y \rightarrow Z$ be the nef value morphism of $(Y, \mathcal{L})$. By using $[$ BFS, Th. 1.1 and $(0.11)]$ we conclude that $Y$ is either

a) a positive defect Fano manifold with Picard group isomorphic to $\mathbb{Z}$, or

b) $\Phi: Y \rightarrow Z$ is a fibration over a normal projective variety $Z$, whose general fibre is a positive defect Fano manifold with Picard group isomorphic to $\mathbb{Z}$.

By Proposition (2.5) only case b) is possible. Moreover, due to Proposition (2.4), $B$ has to contain all fibres of $\Phi$ that it meets. Hence it is contained in a union of fibres of $\Phi$. In particular, the general fibre $F$ of $\Phi: Y \rightarrow Z$ does not meet the branch locus $B$, hence the same happens for the general contact locus $\Lambda \subset F$. But $\Lambda \cong \mathbb{P}^{k}$, where, by BFS, Th. 1.2],

$$
k=\operatorname{def}\left(F,\left.\mathcal{L}\right|_{F}\right)=\operatorname{def}(Y, \mathcal{V})+\operatorname{dim} Z>\operatorname{def}(Y, \mathcal{V})>0 .
$$

So $\Lambda$ cannot admit non-trivial unbranched covers. Hence $\pi^{-1}(\Lambda)=\bigcup_{i=1}^{m} \widetilde{\Lambda_{i}}$, a disjoint union, with $\widetilde{\Lambda_{i}} \cong \Lambda$ via $\pi$, for every $i=1, \ldots, m$. Note that $\left(\left.L\right|_{\widetilde{\Lambda_{i}}}\right)^{k}=$ $\frac{1}{m}\left(\left.\pi^{*} \mathcal{L}\right|_{\Lambda}\right)^{k}=1$, for every $i$. So $(X, L)$ itself is covered by linear $\mathbb{P}^{k}$ 's. Moreover $\pi$ induces an isomorphism $\mathcal{N}_{\widetilde{\Lambda_{i} / X}} \cong \mathcal{N}_{\Lambda / Y}$ for every $i$. It follows that for every $i$, there is a line $\lambda$ of $(X, L)$ such that $\lambda \subset \widetilde{\Lambda_{i}}$ and $\pi(\lambda)=\ell$, a line of $(Y, \mathcal{L})$ contained in $\Lambda$. Taking into account the isomorphism $\mathcal{N}_{\widetilde{\Lambda_{i}} / X} \cong \mathcal{N}_{\Lambda / Y}$ induced by $\pi$, and the relation $K_{\widetilde{\Lambda_{i}}}=\left.K_{X}\right|_{\widetilde{\Lambda_{i}}}+\operatorname{det} \mathcal{N}_{\widetilde{\Lambda_{i}} / X}$ provided by adjunction, we get

$$
-K_{X} \lambda=\operatorname{deg}\left(\left.\mathcal{N}_{\widetilde{\Lambda_{i}} / X}\right|_{\lambda}\right)+\left(\operatorname{dim} \widetilde{\Lambda_{i}}+1\right)=\operatorname{deg}\left(\left.\mathcal{N}_{\Lambda / Y}\right|_{\ell}\right)+(\operatorname{dim} \Lambda+1)=-K_{Y} \ell .
$$

This implies, by BSW, Th. 2.3 and Cor. 2.4], that the nef value of $(X, L)$ is exactly $-K_{Y} \ell=-K_{X} \lambda$. Then the nef value morphism of $(X, L)$ contracts $\widetilde{\Lambda_{i}}$, and hence every connected component of $\pi^{-1}(F)$ is swept out by these linear spaces. This leads to the following commutative diagram:

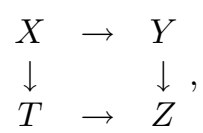

the second vertical arrow being $\Phi: Y \rightarrow Z$ and the first one being the nef value morphism of $(X, L)$. Now recall that every Fano manifold is simply connected $([\mathrm{C}]$, KMM $]$. So, since $F$ is simply connected, we have $\pi^{-1}(F)=\bigcup_{i=1}^{m} \widetilde{F}_{i}$, a disjoint union, with $\widetilde{F}_{i} \cong F$ via $\pi$, for every $i=1, \ldots, m$. This shows that the horizontal morphism $T \rightarrow Z$ in the diagram above is (generically finite) of degree $m$.

This gives the following structure theorem.

(2.6) Theorem. Let $\pi: X \rightarrow Y$ be a branched cyclic cover of degree $m \geq 2$, let $\mathcal{L}$, $L, V$ be as in Section (2.1), and let $B$ be the branch divisor on $Y$. If $\operatorname{def}(X, V)>0$, then $Y$ is a Fano fibration over a normal variety $Z$, via the nef value morphism $\Phi: Y \rightarrow Z, B=\Phi^{*} D$ for some (Weil) divisor $D$ on $Z$, and the nef value morphism $X \rightarrow T$ of $(X, L)$ is also a Fano fibration fitting into a commutative diagram (2.1.3), where the morphism $T \rightarrow Z$ has degree $m$. In particular $(X, L)$ is covered by linear $\mathbb{P}^{k}$ 's, where $k=\operatorname{def}_{0}(X, V)+\operatorname{dim} Z>\operatorname{def}_{0}(X, V)$.

Recalling (2.1.1) and (2.1.2) we see that $k \geq \operatorname{def}(X, V)$. So, the conjecture in LPS1, (2.11)] is satisfied for the special pairs $(X, V)$ considered in this section. 
Here are some examples.

(2.7) Examples. 1) Let $\Pi: W \rightarrow Z$ be a branched cyclic cover of a smooth curve $Z$. For any $r \geq 2$ take the products $X=W \times \mathbb{P}^{r}, Y=Z \times \mathbb{P}^{r}$, a very ample line bundle $\mathcal{L}$ on $Y$ making $(Y, \mathcal{L})$ a scroll over $Z$, and the induced cyclic cover $\pi=(\Pi \times I d): X \rightarrow Y$. Here $\Phi: Y \rightarrow Z$ is the projection onto the first factor, and $B$ consists of a finite number of fibres of $\Phi$.

2) Let $\Pi: S \rightarrow \mathbb{P}^{2}$ be a branched cyclic cover. Let $X=S \times \mathbb{P}^{4}, Y=\mathbb{P}^{2} \times \mathbb{P}^{4}$, and consider the composition $f: X \rightarrow Y \hookrightarrow \mathbb{P}^{14}$ of the map $\pi:=\Pi \times I d: S \times \mathbb{P}^{4} \rightarrow$ $\mathbb{P}^{2} \times \mathbb{P}^{4}$ with the Segre embedding of $\mathbb{P}^{2} \times \mathbb{P}^{4}$. Let $L=f^{*}\left(\mathcal{O}_{\mathbb{P}^{14}}(1)\right)$. In this case $Y$ is a scroll over a surface and $B$ is a scroll over a curve. So both have positive defect (2 and 3 , respectively). Then $\operatorname{def}(X, L)=2$.

In the discussion leading to Theorem (2.6), the integer $k=\operatorname{def}\left(F,\left.\mathcal{L}\right|_{F}\right)$ plays a crucial role. In fact we can prove

(2.8) Proposition. Let things be as in Theorem (2.6); then $k \geq \operatorname{def}_{1}(X, V)=$ $\operatorname{def}_{0}(X, V)+1$.

Proof. Set $k_{0}:=\operatorname{def}_{0}(X, V), k_{1}:=\operatorname{def}_{1}(X, V)$, and recall that $k_{0}$ is the classical defect of $\phi_{\mathcal{V}}(Y) \subset \mathbb{P}^{N}$, as well as $k_{1}$ is the classical defect of an appropriate component, say $D$, of $\phi_{\mathcal{V}}(B)$. As a consequence of Proposition (2.4) and BFS] we know the following facts:

(i) Any contact locus of $\phi_{\mathcal{V}}(Y)$ with a tangent hyperplane is contained in a fibre of the nef value morphism $\Phi: Y \rightarrow Z$.

(ii) $D$ contains any fibre of $\Phi$ that it meets.

Now take a general $b \in D$. Consider a hyperplane $H$ tangent to $\phi_{\mathcal{V}}(Y)$ at $b$. Then we can choose a linear space $\Lambda$ through $b$ of dimension $k_{0}$ such that $H$ is tangent to $\phi_{\mathcal{V}}(Y)$ along $\Lambda$. By (i) and (ii) we get $\Lambda \subset D$. Moreover, for the normal bundle $\mathcal{N}_{D / Y} \cong \mathcal{O}_{D}(D)$, we have $\left.\mathcal{N}_{D / Y}\right|_{\Lambda}=\mathcal{O}_{\Lambda}$, hence $\left.K_{D}\right|_{\Lambda}=\left.K_{Y}\right|_{\Lambda}$. By [BFS, (0.8)] and the previous equality we get $\left.\left(K_{D}+\left(\left(\operatorname{dim}(Y)+k_{0}\right) / 2+1\right) \mathcal{L}\right)\right|_{\Lambda}=\mathcal{O}_{\Lambda}$. This means that the nef value of $\left(D,\left.\mathcal{L}\right|_{D}\right)$ is greater than or equal to $\left(\operatorname{dim}(Y)+k_{0}\right) / 2+1$. Now, use BFS, (0.12)] to show that $\left(\operatorname{dim}(B)+k_{1}\right) / 2+1 \geq\left(\operatorname{dim}(Y)+k_{0}\right) / 2+1$, or equivalently,

$$
k_{1}-1 \geq k_{0} .
$$

On the other hand, the general tangent hyperplane to $D$ is singular along a linear space $\Lambda$ of dimension $k_{1}$ such that $\left.\left(K_{D}+\left(\left(\operatorname{dim}(B)+k_{1}\right) / 2+1\right) \mathcal{L}\right)\right|_{\Lambda}=\mathcal{O}_{\Lambda}$. This implies $\left.\left(K_{Y}+\left(\left(\operatorname{dim}(Y)-1+k_{1}\right) / 2+1\right) \mathcal{L}\right)\right|_{\Lambda}=\mathcal{O}_{\Lambda}$. Then the nef value of $(Y, \mathcal{L})$ is bigger than or equal to $\left(\operatorname{dim}(Y)-1+k_{1}\right) / 2+1$. This means $\left(\operatorname{dim}(Y)+k_{0}\right) / 2+1 \geq$ $\left(\operatorname{dim}(Y)-1+k_{1}\right) / 2+1$ or, equivalently,

$$
k_{0} \geq k_{1}-1 \text {. }
$$

Combining (2.8.1) with (2.8.2) we get $k_{0}=k_{1}-1$, and taking into account (2.1.2), this gives $k=k_{0}+\operatorname{dim}(Z) \geq k_{0}+1=k_{1}$.

\section{Some CONSIDERATIONS ON THE ZERO DEFECT}

Consider $(X, L, V)$ as in Section (0.0). We know by Section (0.0.2) that $\operatorname{def}(X, V)$ $\leq n$ with equality if and only if $(X, L, V)=\left(\mathbb{P}^{n}, \mathcal{O}_{\mathbb{P}}(1), H^{0}\left(\mathbb{P}^{n}, \mathcal{O}_{\mathbb{P}}(1)\right)\right)$. If $\phi_{V}(X)$ $=\mathbb{P}^{n}$, then clearly $\operatorname{def}_{0}(X, V)=n$. If $\phi_{V}(X) \neq \mathbb{P}^{n}$ (not linear in the following), then 
we know that $\operatorname{dim}(\mathcal{D}(X, V)) \geq \operatorname{dim}\left(\mathcal{D}_{0}\right)=N-1-\operatorname{def}_{0}(X, V)$. Since $\operatorname{def}_{0}(X, V)$ is precisely the classical dual defect of $\phi_{V}(X) \subset \mathbb{P}^{N}$, then $\operatorname{def}_{0}(X, V) \leq n-1$. Then, except for the case in which $\phi_{V}(X)$ is linear, we get

$$
\operatorname{def}_{0}(X, V) \leq n-1 .
$$

Example (0.2) shows that (3.0.0) is sharp. This implies

(3.1) Second Bound Lemma. Let $(X, L, V)$ be as in Section (0.0), with $\phi_{V}(X)$ not linear. Then $\operatorname{dim}(\mathcal{D}(X, V)) \geq N-n$.

In fact, this bound is not sharp. In [LPS1, (2.8)] it is shown that if $(X, L) \neq$ $\left(\mathbb{P}^{n}, \mathcal{O}_{\mathbb{P}^{n}}(1)\right)$, then $\operatorname{def}(X, L) \leq n-2$, that is, $\operatorname{dim}(\mathcal{D})=N-1-\operatorname{def}(X, L) \geq N-n+1$. In connection with (3.0.0) it is natural to ask the following.

(3.1.1) Question. What are the possible values of $\operatorname{def}_{0}(X, V)$ for a triplet $(X, L, V)$ as in Section (0.0)?

The following examples answer this question. Moreover they have some relevance in connection with [LPS2] and are significant for the comments in Section (1.12).

(3.2) Example. Consider an irreducible non-degenerate smooth curve $C \subset \mathbb{P}^{N}$. Let $\mathcal{X} \subset C \times \mathbb{P}^{N \vee}$ be the conormal variety and consider the morphisms $p: \mathcal{X} \rightarrow C$, $\pi: \mathcal{X} \rightarrow \mathbb{P}^{N \vee}$ induced by the projections of $C \times \mathbb{P}^{N \vee}$. The former makes $\mathcal{X}$ a $\mathbb{P}^{N-2}$-bundle over $C$, while the image of the latter is, by definition, $C^{\vee}$, the dual variety of $C$. In fact $\mathcal{X}=\mathbb{P}\left(\mathcal{N}_{C / \mathbb{P}^{N}}(-1)\right)$. Clearly, no tangent plane to $C$ can have a positive-dimensional contact locus; moreover $C^{\vee}$ is a hypersurface in $\mathbb{P}^{N \vee}$. Let $\mathcal{L}=\pi^{*} \mathcal{O}_{\mathbb{P}^{N \vee}}(1)$ and set $V=\pi^{*} H^{0}\left(\mathbb{P}^{N \vee}, \mathcal{O}_{\mathbb{P}^{N \vee}}(1)\right)$. Then $\mathcal{L}$ is spanned by $V$, and $\pi=\phi_{V}$ is generically one-to-one. Moreover, since there are no positivedimensional contact loci, the map $\pi$ is finite. It means that $\mathcal{L}$ is ample (equivalently $\mathcal{N}_{C / \mathbb{P}^{N}}(-1)$ is ample). So $(\mathcal{X}, \mathcal{L}, V)$ is a triplet as in Section $(0.0)$, also verifying that the corresponding map is generically one-to-one, exactly as in LPS2, (2.0.1)]. Now look at the discriminant locus $\mathcal{D}(\mathcal{X}, V)$. Recall that the general element of the main component $\mathcal{D}_{0}(\mathcal{X}, V)$ is of the form $A=\pi^{*} h$, where $h$ is a tangent hyperplane to $C^{\vee}$ LPS2, Rem. 2.1]. Thus, under the identification of $|V|$ with $\mathbb{P}^{N}=\left(\mathbb{P}^{N \vee}\right)^{\vee}, \mathcal{D}_{0}(\mathcal{X}, V)$ can be identified with $C=\left(C^{\vee}\right)^{\vee}$. Hence $\operatorname{def}_{0}(\mathcal{X}, V)=$ $N-2=\operatorname{dim}(\mathcal{X})-1$. So, for the general $A \in \mathcal{D}_{0}(\mathcal{X}, V)$ we have that $\operatorname{Sing}(A)$ is an $(N-2)$-plane of $(\mathcal{X}, \mathcal{L})$, in fact a fibre of $p$. It is sent by $\pi$ to a $(N-2)$-plane $P \subset C^{\vee}$ whose points parameterize the hyperplanes of $\mathbb{P}^{N}$ tangent to $C$ at a given point $x$. Since $C^{\vee}$ is a developable variety, any hyperplane $h$ of $\mathbb{P}^{N \vee}$ tangent to $C^{\vee}$ at a point of $P$ is tangent along the whole $P$ (the contact locus of $h$ ). On the other hand, for any point $x \in C$ the linear system of hyperplanes tangent to $C$ at $x$ contains a codimension 1 linear subsystem of osculating hyperplanes; they define, by biduality, a hyperplane $\bar{x} \subset P$, along which $C^{\vee}$ is singular. Hence any hyperplane tangent to $C^{\vee}$ at a general point of $P$ has singularities along $\bar{x}$ worse than non-degenerate quadratic singularities. Coming back to the projective bundle $\mathcal{X}$, this says that there is a divisor $\widetilde{C}$ of $\mathcal{X}$ along which the differential $d \pi$ drops rank; moreover, for every $A \in \mathcal{D}_{0}(\mathcal{X}, V)$ we have that the intersection $\operatorname{Sing}(A) \cap \widetilde{C}$ is non-empty.

This shows the existence of triplets $(X, L, V)$ as in Section (0.0), satisfying additionally that $\phi_{V}$ is generically one-to-one, as in [LPS2, (2.0.1)], for which the equality $\operatorname{def}_{0}(X, L, V)=\operatorname{dim}(X)-1$ holds. This example can be generalized. 
(3.3) Example. Consider a non-degenerate $n$-dimensional smooth variety $X \subset \mathbb{P}^{N}$ such that the vector bundle $\mathcal{N}_{X / \mathbb{P}^{N}}(-1)$ is ample, e.g., a complete intersection. Just as in Example (3.2) take the conormal variety $\mathcal{X}=\mathbb{P}\left(\mathcal{N}_{X / \mathbb{P}^{N}}(-1)\right)$ and the corresponding projections. With the same notation of Example (3.2) we have that $\mathcal{D}_{0}(\mathcal{X}, V)=\left(X^{\vee}\right)^{\vee}=X$. This means that $\operatorname{def}_{0}(\mathcal{X}, V)=N-1-n=\operatorname{dim}(\mathcal{X})-n$. Let us point out that in general, for a smooth variety $X \subset \mathbb{P}^{N}, \operatorname{dim}(X) \geq 2$ the map $\pi: \mathcal{X} \rightarrow \mathbb{P}^{N \vee}$ induced on the conormal variety can contract curves. Recall the simple example of $\left(\mathbb{P}^{2}, \mathcal{O}_{\mathbb{P}^{2}}(2)\right)$.

In Example (3.3) we have shown that for $(X, L, V)$ as in Section (0.0), additionally satisfying that $\phi_{V}$ is generically one-to-one, any possible value of $\operatorname{def}_{0}$, $0 \leq \operatorname{def}_{0}(X, V) \leq \operatorname{dim}(X)$, can be reached, answering Question (3.1.1).

\section{EXTREMAL AND QUASI-EXTREMAL CASES FOR THE ZERO DEFECT}

Our general goal of classifying triplets $(X, L, V)$ with small discriminant variety includes the investigation concerning the largest value of $\operatorname{def}_{0}(X, V)$. According to LPS2 let us consider the following conditions.

(4.0) Let $(X, L, V)$ be a triplet as in Section (0.0) such that $\phi_{V}: X \rightarrow \phi_{V}(X) \subset \mathbb{P}^{N}$ is generically one-to-one.

First of all, note that, under the assumption in Section (4.0), if $\phi_{V}(X)$ is linear, then $(X, L, V)=\left(\mathbb{P}^{n}, \mathcal{O}_{\mathbb{P}^{n}}(1), H^{0}\left(\mathbb{P}^{n}, \mathcal{O}_{\mathbb{P}^{n}}(1)\right)\right)$. So, what we said at the beginning of Section 3 implies the first assertion in [LPS2, Th. 3.5]. The next assertions in that statement are not true, as pointed out in [LPS2, Erratum]. In the present section we face the classification of triples as in Section (4.0) in the extremal case $\operatorname{def}_{0}(X, V)=\operatorname{dim}(X)-1$ and the quasi-extremal case $\operatorname{def}_{0}(X, V)=\operatorname{dim}(X)-2$, when $\phi_{V}$ is generically one-to-one. Let us recall the following definitions.

(4.0.0) Definitions. Let $X \subset \mathbb{P}^{N}$ be a projective variety of dimension $n$. The Gauss map of $X$ is the map $\gamma: U \rightarrow G(n, N)$ from the open set $U$ of smooth points of $X$ to the grassmannian $G(n, N)$ which assigns to any point $x \in U$ the projective tangent space of $X$ at $x$. We will say that $X \subset \mathbb{P}^{N}$ is developable if $\operatorname{dim}(\overline{\gamma(U)})<n$, where $\overline{\gamma(U)}$ stands for the closure of $\gamma(U) \subset G(n, N)$.

(4.1) Proposition. Let $(X, L, V)$ be as in Section (4.0). If $\operatorname{def}_{0}(X, V)=n-1>0$, then $\phi_{V}(X)$ is developable.

Proof. Let $T=\mathbb{P}^{n-1}$ be the contact locus of $\phi_{V}(X)$ with a general $H \in\left(\phi_{V}(X)\right)^{\vee}$. For $Z$ an irreducible component of the Hilbert scheme of $(n-1)$-planes of $\phi_{V}(X)$ write $q: \mathcal{H}_{Z} \rightarrow \phi_{V}(X)$ the natural map from $\mathcal{H}_{Z}$, the total space of the Hilbert scheme over $Z$, to $\phi_{V}(X)$. Choose $Z$ such that $q$ is surjective and the corresponding point to $T$, say $t$, verifies $t \in Z$. If $\operatorname{dim}(Z) \geq 2$, then $\phi_{V}(X)$ is a linear space because for two general points $y, y^{\prime} \in \phi_{V}(X)$ the line joining them is contained in $\phi_{V}(X)$. This means $\operatorname{def}_{0}(X, V)=n$, a contradiction. Then, $\operatorname{dim}(Z)=1$. This implies that there is a finite set of $(n-1)$-planes $T_{1}, \ldots, T_{s}$ through the general point $y \in \phi_{V}(X)$. Since the general tangent hyperplane to $\phi_{V}(X)$ at $y$, say $H_{\alpha}$ ( $\alpha$ moving in a open set $U$ of a linear space), is tangent along a $(n-1)$-plane $T_{\alpha}$, we can say that $T_{\alpha}=T_{1}$ for every $\alpha \in U$ (shrinking $U$ if necessary). Hence for the general $y^{\prime} \in T_{1}$ and for $\alpha \in U$ it holds that $T_{\phi_{V}(X), y^{\prime}} \subset H_{\alpha}$ and then $T_{\phi_{V}(X), y}=T_{\phi_{V}(X), y^{\prime}}$. 
(4.1.1) Remark. Let us observe that the argument of the previous paragraph showing that $\phi_{V}(X)$ is developable only relies on the fact that there is a finite set of linear spaces (candidates to be a contact locus) through the general point.

(4.2) Proposition. Let $(X, L, V)$ be as in Section (4.0). If $\operatorname{def}_{0}(X, V)=\operatorname{dim}(X)-$ 1 , then $(X, L)$ is a scroll over a smooth curve.

Proof. Since $\phi_{V}$ is generically one-to-one, then by [LPS2, Th. 2.3 and Cor. 2.5] $(X, L)$ is covered by $\mathbb{P}^{n-1}$ 's. Let $T$ be a general $\mathbb{P}^{n-1}$ contained in $X$. By [LPS2, Lemma 3.1] the normal bundle $\mathcal{N}_{T / X}$ is globally generated at a general point and then $\mathcal{N}_{T / X}=\mathcal{O}_{T}(a)$ with $a$ an integer, $a \geq 0$. If $a>0$, then $h^{0}\left(\mathcal{N}_{T / X}\right)>1$ and $h^{1}\left(\mathcal{N}_{T / X}\right)=0$. Take $y, y^{\prime} \in \phi_{V}(X)$ general points. Since $\phi_{V}$ is generically one-toone, then there exist $x, x^{\prime} \in X$ such that $\phi_{V}(x)=y$ and $\phi_{V}\left(x^{\prime}\right)=y^{\prime}$. But, since $h^{0}\left(\mathcal{N}_{T / X}\right)>1$ and $h^{1}\left(\mathcal{N}_{T / X}\right)=0$, then there exists a $\mathbb{P}^{n-1}$, say $T_{x, x^{\prime}}$ containing both points $x, x^{\prime}$. Since $\left.L\right|_{T_{x, x^{\prime}}}=\mathcal{O}(1)$, then the line joining $y$ and $y^{\prime}$ is contained in $\phi_{V}(X)$. This means $\phi_{V}(X)=\mathbb{P}^{n}$, a contradiction. Therefore $\mathcal{N}_{T / X}=\mathcal{O}_{T}$. By adjunction formula $\left.K_{X}\right|_{T}=K_{T}=\mathcal{O}_{T}(-n)$. Hence $\left.\left(K_{X}+(n-1) L\right)\right|_{T}=\mathcal{O}_{T}(-1)$. Then the nef value of $(X, L)$ is bigger than $\operatorname{dim}(X)-1$ and so we have (II Th. 1.5] and $[\mathrm{BS}$, Th. 11.1.1]) that $(X, L)$ is one of the following: a scroll over a smooth curve, a quadric $\left(Q^{n}, \mathcal{O}_{Q^{n}}(1)\right)$, or the Veronese surface. But in the second case it must be $V=H^{0}\left(Q^{n}, \mathcal{O}_{Q^{n}}(1)\right)$, hence $\operatorname{def}_{0}(X, V)=0$, a contradiction. The third case does not occur because $\left(\mathbb{P}^{2}, \mathcal{O}_{\mathbb{P}^{2}}(2)\right)$ does not contain lines.

(4.2.0) Remark. Let us observe that for $(X, L, V)$ as in Proposition (4.2) $\phi_{V}$ cannot be an embedding; otherwise $\operatorname{def}_{0}(X, V)=\operatorname{def}\left(\phi_{V}(X)\right)=n-2$.

(4.2.1) Examples. In Example (3.2) we have an example of this situation. Let us show another one. Take $C$ a smooth curve. Consider a very ample line bundle $L$ on $C$ and $V=H^{0}(C, L)$ giving an embedding $C \subset \mathbb{P}^{N}$. The vector bundle $J_{1}(L)$ defines a map $f$ from the projective bundle $\mathbb{P}\left(J_{1}(L)\right)$ onto the developable surface $T C$ of tangents to the curve $C$. If $f$ is generically one-to-one (this means that only a single tangent line goes through the general point of the developable surface) we obtain more examples of this situation. Let us show that $J_{1}(L)$ is ample. Since $J_{1}(L)$ is spanned by global sections, if $J_{1}(L)$ is not ample, we must have a quotient $J_{1}(L) \rightarrow \mathcal{O}_{C} \rightarrow 0$. Hence $H^{0}\left(J_{1}(L)^{\vee}\right) \neq 0$, but this contradicts the following fact.

(4.3) Lemma. Let $X$ be a smooth variety of dimension $n$ and let $L$ be an ample line bundle on $X$. If $H^{0}\left(X, J_{1}(L)^{\vee}\right) \neq 0$, then $(X, L)=\left(\mathbb{P}^{n}, \mathcal{O}_{\mathbb{P}^{n}}(1)\right)$.

Proof. Just take the exact sequence

$$
0 \rightarrow-L \rightarrow J_{1}(L)^{\vee} \rightarrow T_{X}(-L) \rightarrow 0
$$

and use the Mori-Sumihiro-Wahl theorem W.

Note that the previous example is effective. Just take $\left(\mathbb{P}^{1}, \mathcal{O}_{\mathbb{P}^{1}}(d)\right)$ with $d>2$.

Now we can face the problem of classifying triplets $(X, L, V)$ as in Section (4.0) in the quasi-extremal case, that is, $\operatorname{def}_{0}(X, V)=n-2>0$. Let us start with the case of threefolds.

(4.4) Proposition. Let $(X, L, V)$ be as in Section (4.0), $\operatorname{dim}(X)=3$ and $\operatorname{def}_{0}(X, V)=1$. Then $(X, L)$ is a scroll either over a smooth curve or over a smooth surface. 
Proof. The polarized variety $(X, L)$ is swept out by lines. Take a general line $T \subset X$. Just as in the proof of Proposition (4.2), $\mathcal{N}_{T / X}=\mathcal{O}_{T}\left(a_{1}\right) \oplus \mathcal{O}_{T}\left(a_{2}\right)$, where $0 \leq a_{1} \leq a_{2}$. If $a_{2}>1$ or $a_{1}=a_{2}=1$, then $h^{0}\left(\mathcal{N}_{T / X}(-1)\right) \geq 2$ and $h^{1}\left(\mathcal{N}_{T / X}(-1)\right)=0$. This means that the dimension of a Hilbert scheme of lines through a general point of $X$ is bigger than or equal to two. This implies $\phi_{V}(X)=$ $\mathbb{P}^{3}$, a contradiction. Therefore either $\mathcal{N}_{T / X}=\mathcal{O}_{T} \oplus \mathcal{O}_{T}(1)$ or $\mathcal{N}_{T / X}=\mathcal{O}_{T} \oplus \mathcal{O}_{T}$.

If $\mathcal{N}_{T / X}=\mathcal{O}_{T} \oplus \mathcal{O}_{T}(1)$, then $\mathcal{O}_{T}(-2)=K_{T}=K_{X} \otimes \bigwedge^{2} \mathcal{N}_{T / X}$. This means $K_{X} T=-3$. Hence $\left(K_{X}+2 L\right) T<0$. We can conclude exactly as in the last part of Proposition (4.2) that $(X, L)$ is a scroll over a smooth curve. Let us observe that in this case $\phi_{V}$ can be an embedding because if $X \subset \mathbb{P}^{N}$ is a scroll over a smooth curve, then $\operatorname{def}_{0}(X, V)=\operatorname{def}(X, V)=n-2$. Moreover, this case is effective.

Let $\mathcal{N}_{T / X}=\mathcal{O}_{T} \oplus \mathcal{O}_{T}$; first, relying on (4.1.1) we can note the following.

(4.4.1) Since there is a finite set of lines through a general point, then $\phi_{V}(X)$ is developable.

Just as before it holds that $\left(K_{X}+2 L\right) T=0$ and $X$ is swept out by the lines $T$.

(4.4.2) If $\left|K_{X}+2 L\right|=\emptyset$, then by $[\underline{\mathrm{S}}$, (0.2)] and [BS, Th. 11.1.1], $(X, L)$ is one of the following: $\left(\mathbb{P}^{3}, \mathcal{O}_{\mathbb{P}^{3}}(1)\right)$, a smooth quadric $\left(Q^{3}, \mathcal{O}_{Q^{3}}(1)\right)$, or a scroll over a smooth curve $C, \pi: X \rightarrow C$. Only the third possibility is, in principle, possible. Since $\mathcal{N}_{T / X}=\mathcal{O}_{T} \oplus \mathcal{O}_{T}, T$ is not contained in a fibre of $\pi$ and hence it is cutting the general fibre of $\pi$. By Section (4.4.1) there exists a $\mathbb{P}^{3}$, say $R$, such that $T_{\phi_{V}}(X), t=R$ for the general $t \in \phi_{V}(T)$. This means $\phi_{V}\left(\pi^{-1}(\pi(t))\right) \subset R$ for the general $t \in \phi_{V}(T)$, hence $\phi_{V}(X)=\mathbb{P}^{3}$, a contradiction.

(4.4.3) If $\left|K_{X}+2 L\right| \neq \emptyset$, then, by $[\underline{\mathrm{S}}$, (0.3)] and [BS, Th. 11.1.1], $(X, L)$ is one of the following:

(4.4.4) a scroll over a smooth surface,

(4.4.5) a Del Pezzo threefold, that is, $K_{X}=-2 L$,

(4.4.6) a hyperquadric fibration over a smooth curve $C$, that is, there exists a surjective morphism $\Phi: X \rightarrow C$ and an ample line bundle $\mathcal{L}$ on $C$ such that $K_{X}+2 L=\Phi^{*} \mathcal{L}$. In particular, the general fibre $Q$ of $\Phi$ is a smooth quadric and $\left.L\right|_{Q}=\mathcal{O}_{Q}(1)$, then $\left.\phi_{V}\right|_{Q}$ is an isomorphism.

(4.4.4) This case is effective, as pointed out in Example (3.2).

(4.4.5) Del Pezzo threefolds. Under the assumption in Section (4.0), if $(X, L)$ is a Del Pezzo manifold, it follows from the Iskovskih-Fujita classification [F, Th. $8.11]$ that $L$ is very ample. We claim that

$$
V \neq H^{0}(X, L) \text {. }
$$

In fact, if equality holds, then $X$ embedded by $|L|$ is a smooth threefold with defect 1. This implies that $(X, L)$ is a scroll over a smooth curve. Take a general fibre $T$. Since $T$ is embedded linearly we obtain $K_{T}=\left.K_{X}\right|_{T}=-\left.2 L\right|_{T}=\mathcal{O}_{T}(-2)$, a contradiction.

According to the classification [F, Th. 8.11] we have to consider seven possibilities:

a) $(X, L)$ is a cubic hypersurface of $\mathbb{P}^{4}$; but this contradicts (4.4.7).

b) $(X, L)$ is a complete intersection of two quadrics of $\mathbb{P}^{5}$. By (4.4.7) $\phi_{V}(X) \subset \mathbb{P}^{4}$ is a quartic hypersurface obtained as a projection from a point $p \in \mathbb{P}^{5} \backslash X$ of a $(2,2)$ complete intersection of $\mathbb{P}^{5}$. Since $\phi_{V}(X) \subset \mathbb{P}^{4}$ has positive defect, for the general $x \in \phi_{V}(X)$ and the general tangent hyperplane $H^{\prime}$ to $\phi_{V}(X)$ at $x$, there is a line in 
$\phi_{V}(X)$ which is the contact locus of $\phi_{V}(X)$ with $H^{\prime}$. This means that the contact locus of the hyperplane $H=\left\langle H^{\prime}, p\right\rangle$ with $X$ has positive dimension. But this contradicts the ampleness of the normal bundle $\mathcal{N}_{X / \mathbb{P}^{5}}(-1)$.

c) $(X, L)=\left(\mathbb{P}^{1} \times \mathbb{P}^{1} \times \mathbb{P}^{1}, \mathcal{L}_{1}+\mathcal{L}_{2}+\mathcal{L}_{3}\right)$, where $\mathcal{L}_{i}=p_{i}^{*}\left(\mathcal{O}_{\mathbb{P}^{1}}(1)\right), p_{i}: X \rightarrow \mathbb{P}^{1}$ being the projection to the $i$-th factor, $i=1,2,3$. Consider a general element of $\phi_{V}(X)^{\vee}$ and its singular locus $T \subset \phi_{V}(X)$, which is a line. Since $\left.\phi_{V}\right|_{\phi_{V}^{-1}(T)}$ is an isomorphism, we write $T$ instead of $\phi_{V}^{-1}(T)$ with a little abuse of notation. Since $\mathcal{N}_{T / X}$ is trivial, there exists $\rho: X \rightarrow \mathbb{P}^{1}$ (one of the $p_{i}$ 's) such that $T$ is contained in a general fiber of $\rho$ which is a smooth quadric $Q$ for which $\left.L\right|_{Q}=\mathcal{O}_{Q}(1)$ and $\left.\phi_{V}\right|_{Q}$ is an isomorphism. We made the same abuse of notation as before writing $Q$ instead of $\phi_{V}(Q)$. By Section (4.4.1) there exists a $\mathbb{P}^{3}$, say $R$, such that $T_{\phi_{V}(X), t}=R$ for $t$ general in $T$. In particular, since $T \subset Q, T_{Q, t} \subset R$ for $t$ general in $T$ and hence $\langle Q\rangle=R=T_{\phi_{V}(X), t}$. Consider $y$ a general point in $Q$. The same argument as before shows that $\langle Q\rangle=T_{\phi_{V}(X), y}$. This implies that for a general $z \in \phi_{V}(X)$ the general tangent hyperplane to $\phi_{V}(X)$ at $z$ is tangent along a smooth quadric, a contradiction.

d) $(X, L)=\left(\mathbb{P}^{3}, \mathcal{O}_{\mathbb{P}^{3}}(2)\right)$ does not occur since there are no lines in $X$.

e) $(X, L)$ is a smooth linear section of the Plücker embedding of $G(1,4)$, the grassmannian of lines of $\mathbb{P}^{4}$. In $[\mathrm{MP}$, Cor. 1.6] it is shown that the dual of a projection of $X$ to $\mathbb{P}^{4}$ is a hypersurface. Consider $Y \subset \mathbb{P}^{5}$, a projection of $X \subset \mathbb{P}^{6}$ in $\mathbb{P}^{5}$, and let $Y^{\prime} \subset \mathbb{P}^{4}$ be a projection of $Y$ in $\mathbb{P}^{4}$. Since $\left(Y^{\prime}\right)^{\vee} \subset \mathbb{P}^{4 \vee}$ is contained in a hyperplane section of $Y^{\vee} \subset \mathbb{P}^{5 \vee}$ then $Y^{\vee}$ is a hypersurface.

f) $(X, L)=\left(\mathbb{P}\left(T_{\mathbb{P}^{2}}\right), \mathcal{O}(1)\right)$. In fact this is a hyperplane section of the Segre embedding of $\mathbb{P}^{2} \times \mathbb{P}^{2}$ in $\mathbb{P}^{8}$. We can again use $[\mathrm{MP}$, Cor. 1.6] as in e) to exclude this possibility.

g) $(X, L)=\left(B_{p}\left(\mathbb{P}^{3}\right), \pi^{*} \mathcal{O}_{\mathbb{P}^{3}}(2) \otimes E^{-1}\right)$, where $\pi: B_{p}\left(\mathbb{P}^{3}\right) \rightarrow \mathbb{P}^{3}$ is the blowing up of $\mathbb{P}^{3}$ at a point $p \in \mathbb{P}^{3}$ and $E$ is the exceptional divisor. Then $(X, L)$ is a scroll over $\mathbb{P}^{2}$, of which the plane $E$ is a section. Identify $|L|$ with the $\mathbb{P}^{8}=\mathbb{P}\left(H^{0}\left(\mathcal{O}_{\mathbb{P}^{3}}(2) \otimes \mathcal{J}_{p}\right)\right)$ and consider $|V|$ as a linear subspace of the latter. The singular elements of $|L|$ define a hypersurface $D$ of degree 4 . Inside $D$ we have a subvariety $D^{\prime}$ of dimension 5 whose points correspond to the reducible quadrics through $p$. The elements of $|L|$ such that their singular locus is a line corresponds to pairs of planes, both containing $p$. Then they define a variety $D^{\prime \prime} \subset D^{\prime}$ of dimension 4 . It is a local computation to check that it is not possible to choose $V \subset H^{0}(X, L)$ such that $\mathcal{D}_{0}(X, V) \subseteq D^{\prime \prime}$.

(4.4.6) Hyperquadric fibrations. Note that for the general contact locus $T$ of $\phi_{V}(X)$, the line $\phi_{V}^{-1}(T)$ is contained in a smooth fibre of $\Phi$. Hence this case can be excluded by exactly the same arguments as in (c), $\Phi$ playing the same role as $\rho$.

(4.5) Proposition. Let $(X, L, V)$ be as in Section (4.0) with $\operatorname{def}_{0}(X, V)=n-2>$ 0 . Then $(X, L)$ is a scroll either over a smooth curve or over a smooth surface.

Proof. The case $n=3$ is done in Proposition (4.4). If $n>3$ we can argue by induction. Since $L$ is ample and spanned, then, by Bertini's Theorem, the general $X_{1} \in|V|$ is smooth. Now we can take $\left(X_{1}, L_{1}=\left.L\right|_{X_{1}}, V_{1}=\operatorname{Im}\left(V \rightarrow H^{0}\left(L_{1}\right)\right)\right)$ in the same conditions as $(X, L, V)$. In fact, $\operatorname{def}_{0}\left(X_{1}, V_{1}\right)=\operatorname{dim}\left(X_{1}\right)-2$ just because $\phi_{V_{1}}\left(X_{1}\right)$ is a general hyperplane section of $\phi_{V}(X)$. Then, by induction, $\left(X_{1}, L_{1}\right)$ is either a scroll over a curve or over a surface. By [BS, 5.5] if $(X, L)$ is not a scroll over 
a curve or a surface, then either $(X, L)=\left(\mathbb{P}^{3}, \mathcal{O}_{\mathbb{P}^{3}}(2)\right)$ or $X_{1}=\mathbb{P}^{1} \times \mathbb{P}^{2}$, the scroll structure being given by the second projection. The first possibility is excluded in d). For the second one, recall that, in the proof of Proposition (4.4) the scrolls over surfaces appear when the normal bundle to the general contact locus is trivial. This means that the contact loci are fibres of the second projection $\pi_{2}: \mathbb{P}^{1} \times \mathbb{P}^{2} \rightarrow \mathbb{P}^{2}$. But this is a contradiction because the dual variety of $\mathbb{P}^{1} \times \mathbb{P}^{2} \subset \mathbb{P}^{5}$ is smooth and any contact locus is contained in a fibre of the first projection $\pi_{1}: \mathbb{P}^{1} \times \mathbb{P}^{2} \rightarrow \mathbb{P}^{1}$.

Since $\operatorname{def}(X, V) \leq \operatorname{def}_{0}(X, V)$ in particular, we obtain the following.

(4.6) Corollary. Let $(X, L, V)$ be as in Section (4.0) with $\operatorname{def}(X, V)=n-2>0$. Then $(X, L)$ is a scroll either over a smooth curve or over a smooth surface.

\section{REFERENCES}

[BFS] M. C. Beltrametti, M. L. Fania, and A. J. Sommese, On the discriminant variety of a projective manifold, Forum Math. 4 (1992), 529-547. MR.1189013 (93k:14049)

[BS] M. C. Beltrametti and A. J. Sommese, The Adjunction Theory of Complex Projective Varieties, De Gruyter Expositions in Math., vol. 16, De Gruyter, 1995. MR.1318687 (96f:14004)

[BSW] M. C. Beltrametti, A. J. Sommese, and J. A. Wiśniewski, Results on varieties with many lines and their applications to adjunction theory, Complex Algebraic Varieties, Proc. Bayreuth 1990, Lect. Notes in Math, vol. 1507, Springer, 1992, pp. 16-38. MR1178717 (93i:14007)

[C] F. Campana, Connexité rationelle des variétés de Fano, Ann. Sci. Ec. Norm. Sup. 25 (1992), 539-545. MR1191735 (93k:14050)

[E1] L. Ein, Varieties with small dual varieties I, Invent. Math. 86 (1986), 63-74. MR0853445 $(87 \mathrm{~m}: 14047)$

[E2] Varieties with small dual varieties II, Duke Math. J. 52 (1985), 895-907. MR0816391 (87m:14048)

[F] T. Fujita, Classification theories of polarized varieties, London Math. Soc. Lecture Notes Series 155, Cambridge Univ. Press, 1990. MR1162108 (93e:14009)

[I] P. Ionescu, Generalized adjunction and applications, Math. Proc. Camb. Phil. Soc. 99 (1986), 457-472. MR0830359 (87e:14031)

[KMM] J. Kollár, Y. Miyaoka, and S. Mori, Rational connectedness and boundedness of Fano manifolds, J. Diff. Geom. 36 (1992), 765-769. MR1189503 (94g:14021)

[LPS1] A. Lanteri, M. Palleschi, and A. J. Sommese, On the discriminant locus of an ample and spanned line bundle, J. Reine Angew. Math. 477 (1996), 199-219. MR.1405315 (97h:14008)

[LPS2] , Discriminant loci of varieties with smooth normalization, Comm. Algebra 28 (2000), 4179-4200; Erratum, Comm. Algebra 31 (2003), 2027-2028. MR.1772001 (2002a:14007)

[LS1] A. Lanteri and. D. Struppa, Some topological conditions for projective algebraic manifolds with degenerate dual varieties: connections with P-bundles, Rend. Accad. Naz. Lincei (VIII) 77 (1984), 155-158. MR0884367 (88g:14017)

[LS2] - Projective 7-folds with positive defect, Compositio Math. 61 (1987), 329-337. MR.0883486 (88c:14059)

[MP] E. Mezzetti and D. Portelli, On threefolds covered by lines, Abh. Math. Sem. Univ. Hamburg 70 (2000), 211-238. MR.1809546 (2001k:14074)

[M1] R. Muñoz, Varieties with low dimensional dual variety, Manuscripta Math. 94 (1997), 427-435. MR:1484636 (99k:14085)

[M2] , Varieties with degenerate dual variety, Forum Math. 13 (2001), 757-779. MR1861248 (2002h:14089)

[M3] Varieties with almost maximal defect, Rend. Istit. Lombardo Accad. Sci. Lett. Rend. A 133 (2000), 103-114. MR 1871882 (2003c:14060)

[S] A. J. Sommese, On the nonemptiness of the adjoint linear system of a hyperplane section of a threefold, J. Reine Angew. Math. 402 (1989), 211-220. MR.1022801 (90j:14011) 
[T] E. A. Tevelev, Projectively dual varieties, J. of Math. Sci. (N.Y.) 117 (2003), 4585-4732. MR.2027446 (2005c:14069)

[W] J. M. Wahl, Cohomological characterizations of $\mathbb{P}^{n}$, Invent. Math. 72 (1983), 315-322. MR0700774 (84h:14024)

[Z] F. L. Zak, Tangents and Secants of Algebraic Varieties, Math. Monographs, vol. 127, Amer. Math. Soc., 1993. MR.1234494 (94i:14053)

Dipartimento di Matematica "F. Enriques", Università di Milano, Via C. Saldini 50, I-20133 Milano, Italy

E-mail address: lanteri@mat.unimi.it

Departamento de Matemáticas y Física aplicadas y Cc. de la Naturaleza, Universidad Rey juan Carlos, C. Tulipán, E-28933 Móstoles Madrid, Spain

E-mail address: roberto.munoz@urjc.es 